TAIWANESE JOURNAL OF MATHEMATICS

Vol. 16, No. 1, pp. 13-45, February 2012

This paper is available online at http://tjm.math.ntu.edu.tw

\title{
THE SEGAL-BARGMANN TRANSFORM FOR COMPACT QUOTIENTS OF SYMMETRIC SPACES OF THE COMPLEX TYPE
}

\author{
Brian C. Hall and Jeffrey J. Mitchell
}

\begin{abstract}
Let $G / K$ be a Riemannian symmetric space of the complex type, meaning that $G$ is complex semisimple and $K$ is a compact real form. Now let $\Gamma$ be a discrete subgroup of $G$ that acts freely and cocompactly on $G / K$. We consider the Segal-Bargmann transform, defined in terms of the heat equation, on the compact quotient $\Gamma \backslash G / K$. We obtain isometry and inversion formulas precisely parallel to the results we obtained previously for globally symmetric spaces of the complex type. Our results are as parallel as possible to the results one has in the dual compact case. Since there is no known Gutzmer formula in this setting, our proofs make use of double coset integrals and a holomorphic change of variable.
\end{abstract}

\section{INTRODUCTION}

\subsection{Segal-Bargmann transforms}

The Segal-Bargmann transform, in the form that we are considering in this paper, consists of applying the heat operator to a function on a certain Riemannian manifold $\mathcal{M}$ and then analytically continuing the result to an appropriate complexification of $\mathcal{M}$. An isometry formula shows that the $L^{2}$ norm of the original function is equal to an appropriate norm on the Segal-Bargmann transform, and an inversion formula shows how to recover the original function from its Segal-Bargmann transform. So far, this program has been carried out for Euclidean and compact Riemannian symmetric spaces $[18,19,52]$ and more recently for noncompact Riemannian symmetric spaces [29, 30, 36, 47], the Heisenberg group [39], and nilmanifolds [40].

The original motivation for this work came from quantum mechanics, in the work of Segal [48-50] and Bargmann [2]. The Segal-Bargmann transform can be viewed as a sort of "phase space wave function" associated to the original "configuration

Received July 16, 2010, accepted September 20, 2010.

Communicated by Jen-Chih Yao.

2010 Mathematics Subject Classification: Primary 22E30; Secondary 81S30, 35K05.

Key words and phrases: Segal-Bargmann transform, Heat operator, Symmetric space, Eigenfunctions. Supported in part by NSF grant DMS-0555862. 
space wave function." Introduction of the phase space wave function allows for several important new constructions, including coherent states, the Berezin-Toeplitz quantization scheme, and the Berezin transform.

On the other hand, one can consider the Segal-Bargmann transform as a geometric study of the heat operator. From this point of view, the question would be to try to characterize the range of the heat operator (for some fixed time $t>0$ ). See [27]. Since applying the heat operator always gives a real-analytic function, it is natural to try to characterize functions in the range in terms of appropriate conditions on their analytic continuations (the isometry formula). Once the range of the heat operator is characterized, the inversion formula is then a formula for computing the backward heat equation as an integral involving the analytic continuation of a function in the range.

\subsection{Compact Lie groups}

We now review briefly the Segal-Bargmann transform for a compact Lie group with a bi-invariant metric, which is a very special case of a compact symmetric space. The main results of this paper are for compact quotients of symmetric spaces of the "complex type." Noncompact symmetric spaces of the complex type are simply the duals (in the usual duality for symmetric spaces) of compact Lie groups. The formulas for the complex case, and compact quotients thereof, are very similar to those for the compact group case, except that we will have to deal with singularities, which do not arise in the compact case.

Let $K$ be a compact Lie group and $K_{\mathbb{C}}$ its complexification. Let $\Delta$ be the Laplacian on $K$ with respect to a bi-invariant metric, taken to be a negative operator, and let $e^{t \Delta / 2}$ be the associated (forward) heat operator. We fix $t>0$ and define the Segal-Bargmann transform for $K$ as the map taking $f \in L^{2}(K)$ to the holomorphic extension to $K_{\mathbb{C}}$ of $e^{t \Delta / 2}(f)$.

For each $x \in K$, the geometric exponential map $\exp _{x}: T_{x}(K) \rightarrow K$ admits an extension to a holomorphic map of the complexified tangent space $T_{x}(K)_{\mathbb{C}}$ into $K_{\mathbb{C}}$. It can be shown (see [52, Sec. 2] and also [42, Sec. 8]) that every point $z \in K_{\mathbb{C}}$ can be expressed uniquely as

$$
z=\exp _{x} i Y, \quad x \in K, Y \in T_{x}(K),
$$

where $\exp _{x} i Y$ is defined by the just-described holomorphic extension. Let $j_{x}$ be the Jacobian of $\exp _{x}$ and let

$$
j_{x}^{\mathrm{nc}}(Y)=j_{x}(i Y) .
$$

The function $j_{x}^{\text {nc }}$ may be thought of as the Jacobian of the exponential mapping for the noncompact symmetric space dual to $K$. (The superscript "nc" stands for "noncompact.") Let $\rho$ denote half the sum (with multiplicities) of the positive restricted roots for $U / K$. Then the main results $[18,19]$ concerning the Segal-Bargmann transform for $K$ may be described as follows. 
Theorem 1. Let $K$ be a compact Lie group with a bi-invariant metric. Then we have the following results.

The isometry formula. Fix $f$ in $L^{2}(K)$ and $t>0$. Then $F:=e^{t \Delta / 2} f$ has a holomorphic extension to $K_{\mathbb{C}}$ satisfying

$$
\begin{aligned}
& \int_{K}|f(x)|^{2} d x \\
= & e^{-|\rho|^{2} t} \int_{x \in K} \int_{Y \in T_{x}(K)}\left|F\left(\exp _{x} i Y\right)\right|^{2} j_{x}^{\mathrm{nc}}(2 Y)^{1 / 2} \frac{e^{-|Y|^{2} / t}}{(\pi t)^{d / 2}} d Y d x .
\end{aligned}
$$

The surjectivity theorem. Given any holomorphic function $F$ on $K_{\mathbb{C}}$ for which the right-hand side of $(1)$ is finite, there exists a unique $f \in L^{2}(K)$ with $\left.F\right|_{K}=$ $e^{t \Delta / 2} f$.

The inversion formula. If $f \in L^{2}(K)$ is sufficiently regular and $F:=e^{t \Delta / 2} f$, then

$$
f(x)=e^{-|\rho|^{2} t / 2} \int_{T_{x}(K)} F\left(\exp _{x} i Y\right) j_{x}^{\mathrm{nc}}(Y)^{1 / 2} \frac{e^{-|Y|^{2} / 2 t}}{(2 \pi t)^{d / 2}} d Y .
$$

Note that we have $e^{-|Y|^{2} / t}$ and $j_{x}^{\text {nc }}(2 Y)$ in the isometry formula but $e^{-|Y|^{2} / 2 t}$ and $j_{x}^{\mathrm{c}}(Y)$ in the inversion formula. The same results for the the Euclidean symmetric space $\mathbb{R}^{d}$ hold, with $j_{x}^{\text {nc }}(Y) \equiv 1$ and $|\rho|=0$. The $\mathbb{R}^{d}$ result is the Segal-Bargmann transform for Euclidean spaces developed by Segal and Bargmann $[2,50]$, with somewhat different normalization conventions. (See [27, 21] for more information).

For general compact symmetric spaces $U / K$, the isometry and inversion formulas developed by Stenzel [52] involve the heat kernel measure on the dual noncompact symmetric space. In the case where $U / K$ is isometric to a compact Lie group with a bi-invariant metric, the dual noncompact symmetric space is of the "complex type," where there is an explicit formula [14] for the heat kernel, accounting for the simple explicit form of Theorem 1.

The group case is also special because of connections to geometric quantization $[12,13,25,35]$ and the quantization of $(1+1)$-dimensional Yang-Mills theory $[6,22,59]$.

\subsection{Quotients of noncompact symmetric spaces of the complex type}

In this paper, we consider a compact quotient of a noncompact Riemannian symmetric space of the "complex type." Suppose $G$ is a connected complex semisimple group and $K$ a maximal compact subgroup of $G$. Then the manifold $G / K$, equipped with a fixed $G$-invariant Riemannian metric, is a noncompact symmetric space of the complex type. Symmetric space of the noncompact type are nothing but the 
noncompact duals, under the usual duality between symmetric spaces of compact and noncompact types, of compact semisimple Lie groups. The simplest example of a symmetric space of the complex type is hyperbolic 3-space.

In $[29,30]$, we have developed a Segal-Bargmann transform for a noncompact symmetric space $G / K$ of the complex type. (See also [27] further discussion of conceptual issues involved.) In the present paper, we wish to extend that theory to a compact quotient $\Gamma \backslash G / K$, where $\Gamma$ is a discrete subgroup of $G$ acting freely and cocompactly on $G / K$. (Examples of such quotients include compact hyperbolic 3-manifolds.) Although the formulas in the quotient case are essentially identical to the formulas for $G / K$ itself, the proofs, particularly of the isometry formula, are different.

At the intuitive level, the results about the Segal-Bargmann transform for $\Gamma \backslash G / K$ (with $G$ complex) should be obtained from the results for the compact group case by dualizing. This means that we should replace $K$ in Theorem 1 with $\Gamma \backslash G / K$ and $j^{\text {nc }}$ with $j^{\mathrm{c}}$, the Jacobian of the exponential map for the compact symmetric space dual to $G / K$. We also replace $|\rho|^{2}$ with $-|\rho|^{2}$ in the exponential factors in front of the integrals, where $|\rho|^{2}$ is related to the scalar curvature, which is positive in the compact case and negative in the noncompact case.

The challenge on the noncompact side (whether for $G / K$ or for $\Gamma \backslash G / K$ ) is to make sense of the dualized formulas. The main difficulty is the appearance of singularities that do not appear on the compact side. If $f$ is a function on $\Gamma \backslash G / K$ and we set $F=e^{t \Delta / 2} f$ for some fixed $t$, then the function

$$
Y \longmapsto F\left(\exp _{x} Y\right), \quad Y \in T_{x}(\Gamma \backslash G / K)
$$

does not admit an entire analytic holomorphic extension in $Y$. Specifically, the function $F\left(\exp _{x}(i Y)\right)$ will develop singularities once $Y$ gets large enough. (By contrast, in the compact case, if $F$ is of the form $F=e^{t \Delta / 2} f$, then $F\left(\exp _{x}(i Y)\right)$ is nonsingular for all $Y$.) To make sense of the isometry formula or the inversion formula for $\Gamma \backslash G / K$, we need a cancellation of singularities.

The inversion formula for $\Gamma \backslash G / K$ is as follows. Let $f$ be a sufficiently smooth function in $L^{2}(\Gamma \backslash G / K)$ and let $F=e^{t \Delta / 2} f$. Then we have

$$
f(x)=\lim _{R \rightarrow \infty} " e^{|\rho|^{2} t / 2} \int_{\substack{Y \in T_{x}(\Gamma \backslash G / K) \\|Y| \leq R}} F\left(\exp _{x} i Y\right) j_{x}^{\mathrm{c}}(Y)^{1 / 2} \frac{e^{-|Y|^{2} / 2 t}}{(2 \pi t)^{d / 2}} d Y .
$$

Here, " $\lim _{R \rightarrow \infty}$ " means the limit as $R$ tends to infinity of the real-analytic extension of the indicated quantity. That is to say, the integral on the right-hand side of (3) is well-defined for all sufficiently small $R$ and admits a real-analytic continuation in $R$ to $(0, \infty)$. The right-hand side of (3) then is equal to the limit as $R$ tends to infinity of this analytic continuation. (See also Stenzel's work [53] for a different sort 
of inversion formula for noncompact symmetric spaces.) Meanwhile, the isometry formula for $\Gamma \backslash G / K$ reads

$$
\begin{aligned}
& \int_{\Gamma \backslash G / K}|f(x)|^{2} d x \\
= & \lim _{R \rightarrow \infty} " e^{|\rho|^{2} t} \int_{x \in \Gamma \backslash G / K} \int_{\substack{Y \in T_{x}(\Gamma \backslash G / K) \\
|Y| \leq R}}\left|F\left(\exp _{x} i Y\right)\right|^{2} j_{x}^{\mathrm{c}}(2 Y)^{1 / 2} \frac{e^{-|Y|^{2} / t}}{(\pi t)^{d / 2}} d Y d x .
\end{aligned}
$$

In both the inversion formula and the isometry formula, there is a cancellation of singularities that allows the real-analytic extension with respect to $R$ to exist, even though $F\left(\exp _{x} i Y\right)$ becomes singular for large $Y$. In the inversion formula, for example, integral on the right-hand side of (3) is unchanged if we average the function $Y \longmapsto F\left(\exp _{x} i Y\right)$ with respect to the action of $K_{x}$, the group of local isometries of $\Gamma \backslash G / K$ fixing $x$. This averaging process cancels many of the singularities in $F\left(\exp _{x} i Y\right)$; the remaining singularities are canceled by the zeros of the function $j^{\mathrm{c}}(Y)$.

Our inversion and isometry formulas for the Segal-Bargmann transform on $\Gamma \backslash G / K$ are the same as the ones developed in $[29,30]$ for $G / K$, except for replacing $G / K$ with $\Gamma \backslash G / K$ in the obvious places in the formulas. (In both cases, still assuming that $G$ is complex!) In the $G / K$ case, our isometry formula does not coincide with the isometry formula developed by B. Krötz, G. Ólafsson, and R. Stanton [36]. The results of [36] have the advantage of working for arbitrary symmetric spaces of the noncompact type (not just the complex case); our results, meanwhile, have the advantage of being more parallel to what one has in the compact case. (See [46] a Segal-Bargmann transform for radial functions on noncompact symmetric spaces. See also [47] for a refinement of the isometry formula in [36], which also differs from the isometry formula of [30] when specialized to the complex case.)

\subsection{Remarks on the methods used}

We conclude this introduction by discussing the methods of proof. At least conceptually, the proof of the inversion formula on $\Gamma \backslash G / K$ should be similar to the proof of the inversion formula on $G / K$. After all, a function $f$ on $\Gamma \backslash G / K$ lifts to a $\Gamma$-invariant function $\tilde{f}$ on $G / K$. To be sure, $\tilde{f}$ is not square-integrable on $G / K$, but this matters little, since the inversion formula involves no integration over the base manifold. As a result, our proof of the inversion formula for $\Gamma \backslash G / K$ is similar to the proof for $G / K$. The key ingredient is an "intertwining formula," specific to the complex case, between the Euclidean and non-Euclidean Laplacians.

In the case of the isometry formula, lifting to $G / K$ is not helpful, since the lack of square-integrability of $\tilde{f}$ prevents us from formulating the isometry "upstairs" on $G / K$. Meanwhile, the Gutzmer-type formula of J. Faraut $[8,9]$, which is the key 
ingredient in the proofs in $[29,30]$ and also in [36], has no analog (so far as we know) on $\Gamma \backslash G / K$. This means that our proof of the isometry formula for $\Gamma \backslash G / K$ must use methods that are completely different from those in the $[29,30]$. Our proof uses a double coset integral along with a holomorphic change of variable to reduce the isometry formula to the inversion formula. This approach parallels one method of establishing the isometry formula in the compact case, in work of Hall [19] and Stenzel [52].

The proof of the holomorphic change of variable (Theorem 8) applies to compact quotients of general symmetric spaces of the noncompact type, not just those of the complex type. Meanwhile, in cases where the relevant singularities can be understood fairly easily (say, the rank-one case or the even-multiplicity case), it may be possible to develop an inversion formula involving integration against a suitably "unwrapped" version of the heat kernel on the dual compact symmetric space. Thus, it may be possible to develop results similar to those of this paper and $[29,30]$ for other noncompact symmetric spaces and compact quotients thereof.

\section{SET-UP}

We begin this section by recalling certain basic facts about symmetric spaces. A standard reference for this material is [32]. We consider a connected complex semisimple group $G$, a fixed maximal compact subgroup $K$ of $G$, and the quotient manifold $G / K$. We will assume, with no loss of generality, that $G$ acts effectively on $G / K$, which is equivalent to assuming that the Lie algebra $\mathfrak{k}$ of $K$ contains no nonzero ideal of $\mathfrak{g}$ and that the center of $G$ is trivial. There is then a unique involution of $G$ whose fixed-point subgroup is $K$. The Lie algebra $\mathfrak{g}$ then decomposes as $\mathfrak{g}=\mathfrak{k}+\mathfrak{p}$, where $\mathfrak{p}$ is the space on which the associated Lie algebra involution acts as $-I$. (Since $G$ is complex, $\mathfrak{p}$ will be equal to $i \mathfrak{k}$.) We now choose on $\mathfrak{p}$ an inner product invariant under the adjoint action of $K$. We consider the manifold $G / K$ and we identify the tangent space at the identity $\operatorname{coset} x_{0}$ with $\mathfrak{p}$. There is then a unique $G$-invariant Riemannian structure on $G / K$ whose restriction to $T_{x_{0}}(G / K)=\mathfrak{p}$ is the chosen Ad- $K$-invariant inner product. The manifold $G / K$, together with a Riemannian structure of this form, is what we will call a noncompact symmetric space of the complex type.

We let $\mathfrak{a}$ be a maximal commutative subspace of $\mathfrak{p}$ and we let $R \subset \mathfrak{a}^{*}$ denote the set of (restricted) roots for the $(\mathfrak{g}, \mathfrak{k})$. We fix a set of positive roots, which we denote by $R^{+}$. We then let $\mathfrak{a}^{+}$denote the closed fundamental Weyl chamber, that is, the set of $Y \in \mathfrak{a}$ such that $\alpha(Y) \geq 0$ for all $\alpha \in R^{+}$. It is known that every element of $\mathfrak{p}$ can be moved into $\mathfrak{a}^{+}$by the adjoint action of $K$.

We will also consider the compact dual to $G / K$. Let $G_{\mathbb{C}}$ be the complexification of $G$, which contains $G$ as a closed subgroup, in which case the Lie algebra of $G_{\mathbb{C}}$ is $\mathfrak{g}_{\mathbb{C}}=\mathfrak{g}+i \mathfrak{g}$. We define $\mathfrak{u}$ to be the subalgebra of $\mathfrak{g}_{\mathbb{C}}$ given by $\mathfrak{u}:=\mathfrak{k}+i \mathfrak{p}$ and we let 
$U$ be the corresponding connected Lie subgroup of $G_{\mathbb{C}}$, which is compact. We then consider the manifold $U / K$. We think of the tangent space at the identity coset in $U / K$ as $i \mathfrak{p}$. The chosen inner product on $\mathfrak{p}$ then determines an inner product on $i \mathfrak{p}$ in the obvious way. There is then a unique $U$-invariant Riemannian structure on $U / K$ whose restriction to the tangent space at the identity coset is this inner product. The manifold $U / K$, with this Riemannian structure, is a simply connected symmetric space of the compact type in the notation of [32], and is called the compact dual of the symmetric space $G / K$. Since $G / K$ is of the complex type, $U / K$ will be isometric to a compact Lie group with a bi-invariant metric.

We then consider a discrete subgroup $\Gamma$ of $G$ with the property that $\Gamma$ acts freely and cocompactly on $G / K$. The action of $\Gamma$ is then automatically properly discontinuous. It is not obvious but true that such subgroups always exist. The manifold $X:=\Gamma \backslash G / K$ is then what we mean by a compact quotient of $G / K$. We let $\pi$ denote the quotient map from $G / K$ to $\Gamma \backslash G / K$; this map is a covering map. Because the action of $\Gamma \subset G$ on $G / K$ is isometric, the metric on $G / K$ descends unambiguously to $X$. In the case that $G / K$ is hyperbolic 3-space, a compact quotient is nothing but a hyperbolic 3-manifold, that is, an orientable closed 3-manifold of constant negative curvature.

For $R>0$, let $T^{R}(X)$ denote the set of pairs $(x, Y)$ in $T(X)$ with $|Y|<R$. Let $S_{R}$ denote the strip in the complex plane given by

$$
S_{R}=\{u+i v \in \mathbb{C}|| v \mid<R\} .
$$

If $\gamma$ is a unit-speed geodesic in $X$, consider the map $\tau: S_{R} \rightarrow T^{R}(X)$ given by

$$
\tau(u+i v)=(\gamma(u), v \dot{\gamma}(u)) .
$$

In the terminology of Lempert and Szbke [43, 54], a complex structure on $T^{R}(X)$ is called "adapted" (to the given metric on $X$ ) if for each geodesic $\gamma$, the map $\tau$ is holomorphic as a map of $S_{R} \subset \mathbb{C}$ into $T^{R}(X)$. Lempert and Szbke show that for any $R>0$ there exists at most one adapted complex structure and that if $R$ is small enough then an adapted complex structure does exist. (These results hold more generally for any compact, real-analytic Riemannian manifold.) The same complex structure was constructed independently, from a different but equivalent point of view, by Guillemin and Stenzel $[16,17]$.

Given $x \in X$, we may consider the geometric exponential map

$$
\exp _{x}: T_{x}(X) \rightarrow X \subset T(X) .
$$

This map can be analytically continued into a holomorphic map of a neighborhood of the identity in the complexified tangent space $T_{x}(X)_{\mathbb{C}}$ into $T(X)$. This analytically continued exponential map satisfies

$$
\exp _{x}(i Y)=(x, Y),
$$


as may easily be verified from the holomorphicity of the map $\tau$.

If $F$ is a real-analytic function on $X$, it will have an analytic continuation, also called $F$, to some $T^{R^{\prime}}(X)$, for some $R^{\prime} \leq R$. In light of (6), we may write the value of $F$ at a point $(x, Y) \in T^{R^{\prime}}(X)$ as $F\left(\exp _{x} i Y\right)$. This notation is suggestive, because we may alternatively consider the map

$$
Y \rightarrow F\left(\exp _{x} Y\right)
$$

as a real-analytic map of $T_{x}(X)$ into $\mathbb{C}$. Then the expression $F\left(\exp _{x} i Y\right)$ may be thought of equivalently as the analytic continuation of $F$ evaluated at the point $\exp _{x}(i Y)=(x, Y)$, or as the analytic continuation of the map (7), evaluated at the point $i Y$.

For each $x \in X$, we have also the Jacobian $j_{x}$ of the exponential map $\exp _{x}$. To compute $j_{x}$, we choose some $\tilde{x} \in G / K$ that maps to $x$ under quotienting by $\Gamma$. Then we choose some $g \in G$ with $g \cdot x_{0}=\tilde{x}$, where $x_{0}$ is the identity coset in $G / K$. The action of $g$ serves to identify $T_{x_{0}}(G / K)$ with $T_{\tilde{x}}(G / K)$, which is then naturally identifiable with $T_{x}(X)$ by the differential of the covering map from $G / K$ to $\Gamma \backslash G / K$. Finally, $T_{x_{0}}(G / K)$ is naturally identifiable with $\mathfrak{p}$. In this way, we obtain an identification of $T_{x}(X)$ with $\mathfrak{p}$. The identification is not unique, but it is unique up to the adjoint action of $K$ on $\mathfrak{p}$. Under any identification of this sort, $j_{x}$ is invariant under the adjoint action of $K$ on $\mathfrak{p}$, and the restriction of $j_{x}$ to $\mathfrak{a} \subset \mathfrak{p}$ is given by

$$
j_{x}(H)=\prod_{\alpha \in R^{+}}\left(\frac{\sinh \alpha(H)}{\alpha(H)}\right)^{2} .
$$

This formula is the same as the formula for the Jacobian of the exponential map on $G / K$ and reflects that in the complex case, all the (restricted) roots for $G / K$ have multiplicity 2.

From this formula, one can verify that the function $j_{x}$ on $T_{x}(X)$ admits an entire holomorphic extension to the complexification of $T_{x}(X)$, which may be identified with $\mathfrak{p}_{\mathbb{C}}$. Now consider the function $j_{x}^{\mathrm{c}}$ on $T_{x}(X)$ given by

$$
j_{x}^{\mathrm{c}}(Y)=j_{x}(i Y) .
$$

Under our identification of $T_{x}(X)$ with $\mathfrak{p}$, we have that $j_{x}^{\mathrm{c}}$ is invariant under the adjoint action of $K$ and its restriction to $\mathfrak{a}$ is given by

$$
j_{x}^{\mathrm{c}}(H)=\prod_{\alpha \in R^{+}}\left(\frac{\sin \alpha(H)}{\alpha(H)}\right)^{2} .
$$

The superscript "c" in the formula reflects that $j_{x}^{\mathrm{c}}$ coincides with the Jacobian of the exponential mapping for the compact symmetric space $U / K$. 
Note that the formula for $j_{x}^{\mathrm{c}}$, under any identification of $T_{x}(X)$ with $\mathfrak{p}$ of the above sort, is independent of $x$. Thus, in a certain sense, $j_{x}^{\mathrm{c}}$ is "the same" function for each $x$, reflecting that any point in $X$ can be mapped to any other point by a local isometry. For example, in the case of a hyperbolic 3-manifold (with an appropriate normalization of the metric), we have $j_{x}^{\mathrm{c}}(Y)=(\sin |Y| /|Y|)^{2}$ for every $x \in X$.

We now let $j_{x}^{\mathrm{c}}(Y)^{1 / 2}$ be (under our identification of $T_{x}(X)$ with $\mathfrak{p}$ ) the Ad- $K$ invariant function whose restriction to $\mathfrak{a}$ is given by

$$
j_{x}^{\mathrm{c}}(H)^{1 / 2}=\prod_{\alpha \in R^{+}} \frac{\sin \alpha(H)}{\alpha(H)} .
$$

Note that $j_{x}^{\mathrm{c}}(Y)^{1 / 2}$ is not the positive square root of $j_{x}^{\mathrm{c}}(Y)$. Rather, $j_{x}^{\mathrm{c}}(Y)^{1 / 2}$ is chosen so as to be real analytic and positive near the origin. We then let $j_{x}^{\mathrm{c}}(Y)^{-1 / 2}$ be the reciprocal of $j_{x}^{\mathrm{c}}(Y)^{1 / 2}$, defined away from the points where $j_{x}^{\mathrm{c}}(Y)$ is zero.

\section{The InVERSION Formula}

The key result of this section is the partial inversion formula (Theorem 3), which is proved using an intertwining formula that relates the Laplacian on $G / K$ to the Euclidean Laplacian. Once Theorem 3 is proved, the desired "global" inversion formulas follow by a fairly straightforward limit as the radius tends to infinity.

For each $x \in \Gamma \backslash G / K$, consider (as in $[29,30]$ ) the function

$$
\nu_{t, x}^{\mathrm{c}}(Y):=e^{t|\rho|^{2} / 2} j_{x}^{\mathrm{c}}(Y)^{-1 / 2} \frac{e^{-|Y|^{2} / 2 t}}{(2 \pi t)^{d / 2}}, \quad Y \in T_{x}(X)
$$

and the associated signed measure

$$
\nu_{t, x}^{\mathrm{c}}(Y) j_{x}^{\mathrm{c}}(Y) d Y=e^{t|\rho|^{2} / 2} j_{x}^{\mathrm{c}}(Y)^{1 / 2} \frac{e^{-|Y|^{2} / 2 t}}{(2 \pi t)^{d / 2}} d Y .
$$

Here, again, the superscript "c" is supposed to denote quantities associated to the compact symmetric space $U / K$ dual to $G / K$. The measure in (9) is an "unwrapped" form of the heat kernel measure on $U / K$. This means that the push-forward of this measure under the exponential mapping for $U / K$ is precisely the heat kernel measure at the identity coset on $U / K[29, \mathrm{Thm}$. 5]. (Note that because $U / K$ is isometric to a compact Lie group with a bi-invariant metric, the heat kernel formula of Ėskin ([7]; see also [58]) applies. From this formula it is easy to see that the signed measure in (9) pushes forward to the heat kernel on $U / K$.)

We introduce the operator

$$
\begin{aligned}
A_{t, R}(f)(x) & =\int_{T_{x}^{R}(X)} F\left(\exp _{x} i Y\right) \nu_{t, x}^{\mathrm{c}}(Y) j_{x}^{\mathrm{c}}(Y) d Y \\
& =e^{t|\rho|^{2} / 2} \int_{T_{x}^{R}(X)} F\left(\exp _{x} i Y\right) j_{x}^{\mathrm{c}}(Y)^{1 / 2} \frac{e^{-|Y|^{2} / 2 t}}{(2 \pi t)^{d / 2}} d Y,
\end{aligned}
$$


where as usual, $F$ is the analytic continuation of $e^{t \Delta / 2} f$ and where $T_{x}^{R}(X)$ denotes the vectors in $T_{x}(X)$ with length less than $R$. The operator $A_{t, R}$ consists of applying the time- $t$ heat operator and then doing a "partial inversion," in which we integrate only over a ball of radius $R$ in the tangent space. We will seek a way to allow $R$ to tend to infinity, by means of an appropriate analytic continuation, with the expectation that $A_{t, R}$ tends to the identity operator as $R$ tends to infinity.

We now state the results of this section, before turning to the proofs.

Proposition 2. There exists $R_{0}>0$ such that for all $f \in L^{2}(X)$, the function $F:=e^{t \Delta / 2} f$ has a holomorphic extension to $T^{R_{0}}(X)$, with respect to the adapted complex structure. Furthermore, for each fixed $z \in T^{R_{0}}(X)$, the map $f \rightarrow F(z)$ is a bounded linear functional on $L^{2}(X)$, with norm a locally bounded function of $z$.

This proposition shows that the operator $A_{t, R}$ is well defined and bounded for all sufficiently small $R$.

Theorem 3. ([Partial Inversion Formula]). Let $R_{0}$ be as in Proposition 2. For all $R<R_{0}$, let $A_{t, R}$ be the operator defined by (10). Then $A_{t, R}$ is a bounded operator on $L^{2}(X)$ and is given by

$$
A_{t, R}=\alpha_{t, R}(-\Delta)
$$

where $\alpha_{t, R}:[0, \infty) \rightarrow \mathbb{R}$ is given by

$$
\alpha_{t, R}(\lambda)=e^{-t \lambda / 2} e^{t|\rho|^{2} / 2} \int_{\substack{Y \in \mathbb{R}^{d} \\|Y| \leq R}} \exp \left(\sqrt{\lambda-|\rho|^{2}} y_{1}\right) \frac{e^{-|Y|^{2} / 2 t}}{(2 \pi t)^{d / 2}} d Y .
$$

Here $\sqrt{\lambda-|\rho|^{2}}$ is either of the two square roots of $\lambda-|\rho|^{2}$.

On $G / K$, the spectrum of $-\Delta$ is the interval $\left[|\rho|^{2}, \infty\right)$. By contrast, on $X=$ $\Gamma \backslash G / K$, the spectrum of $-\Delta$ includes points in the interval $\left[0,|\rho|^{2}\right)$; for example, the constant function 1 is an eigenvector for $-\Delta$ with eigenvalue 0 . For $\lambda \in\left[0,|\rho|^{2}\right)$, $\sqrt{\lambda-|\rho|^{2}}$ will be pure imaginary. Nevertheless, because the domain of integration in (11) is invariant under $y \rightarrow-y$, the value of $\alpha_{t, R}(\lambda)$ is still a real number.

For each fixed value of $R$ and $t$, the integral in (11) is bounded by a constant times $\exp (\sqrt{\lambda} R)$. Thus, because of the factor of $e^{-t \lambda / 2}$ in front of the integral, $\alpha_{t, R}(\lambda)$ is a bounded function of $\lambda$ for each $R$ and $t$.

Although the definition of $A_{t, R}$ in (10) makes sense only for small $R$, the function in (11) is a well defined and bounded function of $\lambda$ for every $R>0$. Furthermore, if we let $R$ tend to infinity in the definition of $\alpha_{t, R}$ we obtain (by 
Dominated Convergence) an integral over all of $\mathbb{R}^{d}$. This integral is an easily evaluated Gaussian integral, whose value turns out to be 1 for all $\lambda$. That is to say,

$$
\lim _{R \rightarrow \infty} \alpha_{t, R}(\lambda)=1
$$

for all $t$ and $\lambda$. This suggests that $A_{t, R}(f)$ should tend to $f$ as $R$ tends to infinity; proving this will yield a global inversion formula. We present two versions of the formula, an $L^{2}$ version valid for all $f \in L^{2}(X)$ and a pointwise version valid for sufficiently smooth $f$.

Theorem 4. ([Global Inversion Formula, $L^{2}$ Version]). Let $R_{0}$ be as in Proposition 2. For all $R<R_{0}$, let $A_{t, R}$ be as in (10). Then the map $R \rightarrow A_{t, R}$ has a weakly analytic extension, also denoted $A_{t, R}$, to a map of $(0, \infty)$ into the space of bounded operators on $L^{2}(X)$. This analytic extension has the property that for each $f \in L^{2}(X)$ we have

$$
f=\lim _{R \rightarrow \infty} A_{t, R} f
$$

with the limit being in the norm topology of $L^{2}(X)$. In light of the original expression for $A_{t, R}$, we may express (13) informally as

$$
f(x)=“ \lim _{R \rightarrow \infty} " e^{t|\rho|^{2} / 2} \int_{T_{x}^{R}(X)} F\left(\exp _{x} i Y\right) j_{x}^{\mathrm{c}}(Y)^{1 / 2} \frac{e^{-|Y|^{2} / 2 t}}{(2 \pi t)^{d / 2}} d Y,
$$

with the limit in the $L^{2}$ sense.

Recall that a map $\alpha$ of $(0, \infty)$ into the space of bounded operators on a Hilbert space $H$ is weakly analytic if the map $R \rightarrow\langle f, \alpha(R) g\rangle$ is a real-analytic function of $R$ for each $f$ and $g$ in $H$. Of course, the analytic extension of the map $R \rightarrow A_{t, R}$ is given by $\alpha_{t, R}(-\Delta)$, where $\alpha_{t, R}$ is defined (for all $R>0$ ) by (11).

Theorem 5. ([Global Inversion Formula, Pointwise Version]). Let $R_{0}$ be as in Proposition 2. For all $R<R_{0}$, let $A_{t, R}$ be as in (10). Assume that $f \in L^{2}(X)$ is in the domain of $\Delta^{l}$ for some positive real number with $l>\left(3 d^{2}-d\right) / 4$. Then for each $x \in X$, the function $L_{x, f}(F)$ given by

$$
L_{x, f}(R)=\left(A_{t, R} f\right)(x)
$$

has a real-analytic extension, also denoted $L_{x, f}$, from $R \in\left(0, R_{0}\right)$ to $R \in(0, \infty)$. Furthermore, we have

$$
f(x)=\lim _{R \rightarrow \infty} L_{x, f}(R)
$$


with the limit being uniform in $x$. In light of the original expression for $A_{t, R}$, we may express (13) informally as

$$
f(x)=“ \lim _{R \rightarrow \infty} " e^{t|\rho|^{2} / 2} \int_{T_{x}^{R}(X)} F\left(\exp _{x} i Y\right) j_{x}^{\mathrm{c}}(Y)^{1 / 2} \frac{e^{-|Y|^{2} / 2 t}}{(2 \pi t)^{d / 2}} d Y,
$$

for $f$ in $\operatorname{Dom}\left(\Delta^{l}\right)$, with the limit being uniform in $x$.

These inversion formulas are as parallel as possible to the inversion formula (2) in the dual compact group case. Specifically, the inversion formulas above are obtained by "dualizing" (2) (changing $j_{x}^{\text {nc }}$ to $j_{x}^{\mathrm{c}}$ and $e^{-t|\rho|^{2} / 2}$ to $e^{t|\rho|^{2} / 2}$ ) and inserting an analytic continuation in $R$, which is unnecessary in the compact group case.

Proof of Proposition 2. Let $k_{t}(\cdot, \cdot)$ denote the heat kernel for $X$. A result of Nelson [44, Thm. 8] shows that for any fixed positive time $t, k_{t}$ is a real-analytic function on $X \times X$. As a result, $k_{t}$ will have an analytic continuation, also denoted $k_{t}$, to $T^{R_{0}}(X) \times T^{R_{0}}(X)$ for some sufficiently small $R_{0}$. Then the function defined by

$$
z \rightarrow \int_{X} k_{t}(z, y) f(y) d y, \quad z \in T^{R_{0}}(X),
$$

is the desired holomorphic extension of $F:=e^{t \Delta / 2} f$. The desired properties of the pointwise evaluation functional are then easy to read off.

Now, Nelson's result leaves open the possibility that the radius $R_{0}$ could depend on $t$, which is harmless in our case, since we work with one fixed $t$ throughout. Nevertheless, using a result of Guillemin and Stenzel [17, Thm. 5.2], it is not hard to see that $R_{0}$ can be chosen to be independent of $t$.

Proof of Theorem 3. Now that $A_{t, R}$ is known to be a bounded operator, we can compute it by evaluating it on an orthonormal basis for $L^{2}(X)$ consisting of eigenfunctions of the Laplacian. So let $\phi$ be an eigenfunction of $-\Delta$ on $X$ with eigenvalue $\lambda \geq 0$. Our goal is to show that $A_{t, R}(\phi)$ is a certain constant multiple of $\phi$, with the constant depending only on $\lambda$. This will show that $A_{t, R}$ is a specific function of the Laplacian.

Applying $e^{t \Delta / 2}$ to $\phi$ gives $e^{-t \lambda / 2} \phi$. This means that we want to compute

$$
e^{-t \lambda / 2} \int_{T_{x}^{R}(X)} \phi\left(\exp _{x} i Y\right) \nu_{t, x}^{\mathrm{c}}(Y) j_{x}^{\mathrm{c}}(Y) d Y,
$$

for a fixed $x$ in $X=\Gamma \backslash G / K$. Let $K_{x}$ denote the identity component of the group of local isometries of $X$ that fix $x$. Then the key point is that the function $\nu_{t, x}^{\mathrm{c}}(Y) j_{x}^{\mathrm{c}}(Y)$ is invariant under the action of $K_{x}$ on $T_{x}(X)$. (This invariance can be seen by observing that under our identification of $T_{x}(X)$ with $\mathfrak{p}, j_{x}^{\mathrm{c}}$ is an Ad- $K$-invariant 
function on $\mathfrak{p}$.) Thus averaging the function $Y \longmapsto \phi\left(\exp _{x} i Y\right)$ over the action of $K_{x}$ has no effect on the integral. This averaging cancels out many of the singularities in $\phi\left(\exp _{x} i Y\right)$.

Let $\tilde{x}$ be a preimage of $x$ in $G / K$ and let $\Phi$ be the lift of $\phi$ to $G / K$. Let $\Phi^{(\tilde{x})}$ denote the radialization of $\Phi$ about $\tilde{x}$, that is, the average of $\Phi$ over the action of $K_{\tilde{x}}$, where $K_{\tilde{x}}$ is the stabilizer of $\tilde{x}$ in $G$. Because $\nu_{t, x}^{\mathrm{c}}(Y) j_{x}^{\mathrm{c}}(Y)$ is invariant under the action of $K_{x}$, we have

$$
\begin{aligned}
& e^{-t \lambda / 2} \int_{T_{x}^{R}(X)} \phi\left(\exp _{x} i Y\right) \nu_{t, x}^{\mathrm{c}}(Y) j_{x}^{\mathrm{c}}(Y) d Y \\
= & e^{-t \lambda / 2} \int_{T_{\tilde{x}}^{R}(G / K)} \Phi^{(\tilde{x})}\left(\exp _{\tilde{x}} i Y\right) \nu_{t, x}^{\mathrm{c}}(Y) j_{x}^{\mathrm{c}}(Y) d Y \\
= & e^{-t \lambda / 2} e^{t|\rho|^{2} / 2} \int_{T_{\tilde{x}}^{R}(G / K)} \Phi^{(\tilde{x})}\left(\exp _{\tilde{x}} i Y\right) j_{x}^{\mathrm{c}}(Y)^{1 / 2} \frac{e^{-|Y|^{2} / 2 t}}{(2 \pi \tau)^{n / 2}} d Y .
\end{aligned}
$$

Now, $\Phi^{(\tilde{x})}$ is a $K_{\tilde{x}}$-invariant eigenfunction for $\Delta$ on $G / K$ with eigenvalue $\lambda$. We now use an "intertwining formula" that relates the Laplacian on $G / K$ to the Euclidean Laplacian on $\mathfrak{p}$, when applied to $K$-invariant functions. See the proof of Theorem 2 in [29] Proposition V.5.1 in [34] and the calculations in the complex case on p. 484. The intertwining formula tells us that the function

$$
Y \rightarrow \Phi^{(\tilde{x})}\left(\exp _{\tilde{x}} Y\right) j_{x}(Y)^{1 / 2}
$$

is an eigenfunction for the Euclidean Laplacian on $\mathfrak{p}$ with eigenvalue $-\left(\lambda-|\rho|^{2}\right)$. Therefore, if we analytically continue and replace $Y$ by $i Y$, we conclude that the function

$$
\Psi(Y):=\Phi^{(\tilde{x})}\left(\exp _{\tilde{x}} i Y\right) j_{x}^{\mathrm{c}}(Y)^{1 / 2}
$$

is an eigenfunction for $\Delta$ on $\mathfrak{p}$ with eigenvalue $\left(\lambda-|\rho|^{2}\right)$. (Recall that $j_{x}^{\mathrm{c}}(Y)=$ $j_{x}(i Y)$.)

We now make use of the following elementary result, which was Lemma 5 of [30]. (We have replaced $2 R$ by $R$ in the statement of [30, Lem. 5].)

Lemma 6. Let $\Psi$ be a smooth function on the ball $B\left(R_{0}, 0\right)$ in $\mathbb{R}^{d}$ satisfying $\Delta \Psi=\sigma \Psi$ for some constant $\sigma \in \mathbb{R}$, where $\Delta$ is the Euclidean Laplacian. Let $\beta$ be a bounded, measurable, rotationally invariant function on $B\left(R_{0}, 0\right)$. Then for all $R<R_{0}$ we have

$$
\int_{|Y| \leq R} \Psi(Y) \beta(Y) d Y=\Psi(0) \int_{|Y| \leq R} e^{\sqrt{\sigma} y_{1}} \beta(Y) d Y .
$$

Here $Y=\left(y_{1}, \ldots, y_{d}\right)$ and $\sqrt{\sigma}$ is either of the two square roots of $\sigma$. 
Lemma 6 therefore tells us that the last line in (15) is equal to

$$
\Phi^{(\tilde{x})}(\tilde{x}) e^{-t \lambda / 2} e^{t|\rho|^{2} / 2} \int_{\substack{Y \in \mathbb{R}^{d} \\|Y| \leq R}} \exp \left(\sqrt{\lambda-|\rho|^{2}} y_{1}\right) \frac{e^{-|Y|^{2} / 2 t}}{(2 \pi \tau)^{d / 2}} d Y .
$$

Since $\Phi^{(\tilde{x})}(\tilde{x})=\Phi(\tilde{x})=\phi(x)$, this establishes that $A_{t, R} \phi=\alpha_{t, R}(-\Delta) \phi$. Since $A_{t, R}$ is known to be bounded and since there exists an orthonormal basis for $L^{2}(X)$ consisting of eigenfunctions of $-\Delta$, the partial inversion formula follows.

We now turn to the proof of the global inversion formula, in two versions. Ultimately, the global inversion formula derives from the partial inversion formula (Theorem 3) together with the observation that $\lim _{R \rightarrow \infty} \alpha_{t, R}(\lambda)=1$ (see (12)).

Proofof Theorem 4. For all $R>0$, we define $A_{t, R}$ to be $\alpha_{t, R}(-\Delta)$, where $\alpha_{t, R}$ is defined by (11). The partial inversion formula (Theorem 3 ) tells us that for $R<R_{0}, A_{t, R}$ coincides with the operator defined in (10). We need to establish, then, that the operator $\alpha_{t, R}(-\Delta)$ is weakly analytic as a function of $R$ for fixed $t$. We choose an orthonormal basis $\left\{\psi_{n}\right\}$ for $L^{2}(X)$ consisting of eigenvectors for $-\Delta$, with corresponding eigenvalues $\lambda_{n}$. Since $-\Delta$ has non-negative discrete spectrum, there is some $N$ with $\lambda_{n} \geq|\rho|^{2}$ for all $n \geq N$. Given $f, g \in L^{2}(X)$, we write $f=\sum a_{n} \psi_{n}$ and $g=\sum b_{n} \psi_{n}$. Then

$$
\left\langle f, \alpha_{t, R}(-\Delta) g\right\rangle_{L^{2}(X)}=\sum_{n=1}^{\infty} \overline{a_{n}} b_{n} \alpha_{t, R}\left(\lambda_{n}\right) .
$$

We use the integral expression (11) for $\alpha_{t, R}$ and we wish to interchange the integral with the sum over $n$. To do this we split off the first $N$ terms and we want to show that Fubini's Theorem applies to the remaining infinite sum. Note that the exponential in the definition of $\alpha_{t, R}$ is positive provided that $\lambda_{n} \geq|\rho|^{2}$. Thus, if we the integral over $|Y| \leq R$ is bounded by the integral over all of $\mathbb{R}^{d}$, which we have already remarked is an easy Gaussian integral (see (12)). Thus, if we put absolute values inside and then interchange the sum and integral, we get an expression that is bounded by

$$
\begin{aligned}
& \sum_{n=N}^{\infty}\left|a_{n}\right|\left|b_{n}\right| e^{-t \lambda_{n} / 2} e^{t|\rho|^{2} / 2} \int_{\mathbb{R}^{d}} \exp \left(\sqrt{\lambda_{n}-|\rho|^{2}} y_{1}\right) \frac{e^{-|Y|^{2} / 2 t}}{(2 \pi t)^{d / 2}} d Y \\
= & \sum_{n=N}^{\infty}\left|a_{n}\right|\left|b_{n}\right| \leq\|f\|_{L^{2}(X)}\|g\|_{L^{2}(X)}<\infty .
\end{aligned}
$$

We may therefore write 


$$
\begin{aligned}
& \left\langle f, \alpha_{t, R}(-\Delta) g\right\rangle_{L^{2}(X)} \\
= & \int_{\substack{Y \in \mathbb{R}^{d} \\
|Y| \leq R}}\left[\sum_{n=1}^{\infty} \overline{a_{n}} b_{n} e^{-t \lambda_{n} / 2} e^{t|\rho|^{2} / 2} \exp \left(\sqrt{\lambda_{n}-|\rho|^{2}} y_{1}\right)\right] \frac{e^{-|Y|^{2} / 2 t}}{(2 \pi t)^{d / 2}} d Y .
\end{aligned}
$$

It is not hard to show, using Fubini's and Morera's Theorems, that the expression in square brackets admits an entire holomorphic extension in $y_{1}$, given by the same formula. From this, it then follows easily that the integral on the right-hand side of (18) is a real-analytic function of $R$. This shows that the operator $\alpha_{t, R}(-\Delta)$ is weakly analytic as a function of $R$, which is therefore (in light of Theorem 3 ) the desired weakly analytic extension of $A_{t, R}$.

Now that we know that the weakly analytic extension of $A_{t, R}$ is given by $\alpha_{t, R}(-\Delta)$, we need to show that $\alpha_{t, R}(-\Delta) f$ tends to $f$ in the norm topology of $L^{2}(X)$, for any $f \in L^{2}(X)$. As above, write $f=\sum a_{n} \psi_{n}$, so that $\alpha_{t, R}(-\Delta) f=$ $\sum_{n=1}^{\infty} a_{n} \alpha_{t, R}\left(\lambda_{n}\right) \psi_{n}$, because $\alpha_{t, R}(-\Delta)$ is bounded. (In both cases, convergence is in $L^{2}(X)$.) Then

$$
\left\|f-\alpha_{t, R}(-\Delta) f\right\|^{2}=\sum_{n=1}^{N}\left(1-\alpha_{t, R}\left(\lambda_{n}\right)\right)^{2}\left|a_{n}\right|^{2}+\sum_{n=N+1}^{\infty}\left(1-\alpha_{t, R}\left(\lambda_{n}\right)\right)^{2}\left|a_{n}\right|^{2},
$$

where again $\lambda_{n} \geq|\rho|^{2}$ for $n>N$. From (11), we can see that $\alpha_{t, R}(\lambda)$ is nonnegative and monotone in $R$ for fixed $t$ and $\lambda$, provided that $\lambda \geq|\rho|^{2}$. Since $\lim _{R \rightarrow \infty} \alpha_{t, R}(\lambda)=1$ (see (12)) this means that $0<\alpha_{t, R}(\lambda) \leq 1$ for $\lambda \geq|\rho|^{2}$. Thus Dominated Convergence shows that the second term on the right-hand side of (19) tends to zero as $R$ tends to infinity. The first term also tends to zero by (12), since it is a finite sum. Thus the left-hand side of (19) tends to zero as $R$ tends to infinity (with $t$ fixed), establishing the $L^{2}$ form of the global inversion formula.

We turn now to the pointwise version of the global inversion formula.

Proof of Theorem 5. As in the previous proof, write $f=\sum_{n=1}^{\infty} a_{n} \psi_{n}$, with convergence in $L^{2}$. We now assume that the eigenvectors $\psi_{n}$ are ordered so that the corresponding eigenvalues are nondecreasing with $n$. According to Weyl's Law, $\lambda_{n}$ behaves asymptotically like a constant times $n^{2 / d}$ as $n$ tends to infinity, where $d=\operatorname{dim} X$, as usual. It is also known (e.g., [51] and the references therein) that there is a constant $C$, depending only on the choice of $X$, such that if $\phi$ is an eigenfunction of the Laplacian with eigenvalue $\lambda$ and normalized to have $L^{2}$ norm 1 , then

$$
\|\phi\|_{L^{\infty}} \leq C_{1} \lambda^{(d-1) / 4}
$$

Thus,

$$
\left\|\psi_{n}\right\|_{L^{\infty}} \leq C_{2}\left(n^{2 / d}\right)^{(d-1) / 4}=C_{2} n^{(d-1) / 2 d} .
$$


On the other hand, if $f$ is in the domain of the $l^{\text {th }}$ power of $\Delta$, then

$$
\sum_{n=1}^{\infty}\left|a_{n}\right|^{2} \lambda_{n}^{2 l}<\infty,
$$

which implies (using Weyl's Law again) that $\left|a_{n}\right| \leq C n^{-2 l / d}$. If, then, $l$ is large enough that

$$
\varepsilon:=\frac{2 l}{d}-\frac{d-1}{2 d}-1>0
$$

(which is equivalent to $l>\left(3 d^{2}-d\right) / 4$ ) we will have

$$
\begin{aligned}
\sum_{n=1}^{\infty}\left|a_{n}\right| \sup \left|\psi_{n}\right| & \leq C_{3} \sum_{n=1}^{\infty} n^{-2 l / d} n^{(d-1) / 2 d} \\
& =C_{3} \sum_{n=1}^{\infty} n^{-(1+\varepsilon)}<\infty
\end{aligned}
$$

Thus by the Weierstrass $M$-test, the series $\sum a_{n} \psi_{n}$ converges uniformly as well as in $L^{2}$ to $f$.

Meanwhile, $\alpha_{t, R}(-\Delta) f=\sum_{n} \alpha_{t, R}\left(\lambda_{n}\right) a_{n} \psi_{n}$, with convergence in $L^{2}$. Since $0 \leq \alpha_{t, R}\left(\lambda_{n}\right) \leq 1$ for $n>N$, this series also converges uniformly (to $\alpha_{t, R}(-\Delta) f$ ). We now plug in the integral formula (11) for $\alpha_{t, R}$ and we wish to interchange (for each fixed $x$ ) the sum over $n$ in $\sum_{n} \alpha_{t, R}\left(\lambda_{n}\right) a_{n} \psi_{n}$ with the integral in (11). To do this, we again split off the terms with $n \leq N$ and argue as in (17) for the applicability of Fubini's Theorem in the remaining terms, substituting the convergence result (21) for $\sum\left|a_{n}\right|\left|b_{n}\right|<\infty$.

We obtain, then,

$$
\begin{aligned}
& \left(\alpha_{t, R}(-\Delta) f\right)(x) \\
= & \sum_{n=1}^{\infty} \alpha_{t, R}\left(\lambda_{n}\right) a_{n} \psi_{n}(x) \\
= & \int_{\substack{Y \in \mathbb{R}^{d} \\
|Y| \leq R}}^{\infty}\left[\sum_{n=1}^{\infty} e^{-t \lambda_{n} / 2} e^{t|\rho|^{2} / 2} \exp \left(\sqrt{\lambda_{n}-|\rho|^{2}} y_{1}\right) a_{n} \psi_{n}(x)\right] \frac{e^{-|Y|^{2} / 2 t}}{(2 \pi t)^{d / 2}} d Y
\end{aligned}
$$

As in the previous proof, the expression in square brackets is an entire function of $y_{1}$ and the whole integral is a real-analytic function of $R$.

Meanwhile

$$
\begin{aligned}
& f(x)-\left(\alpha_{t, R}(-\Delta) f\right)(x) \\
= & \sum_{n=1}^{N}\left(1-\alpha_{t, R}\left(\lambda_{n}\right)\right) a_{n} \psi_{n}(x)+\sum_{n=N+1}^{\infty}\left(1-\alpha_{t, R}\left(\lambda_{n}\right)\right) a_{n} \psi_{n}(x) .
\end{aligned}
$$


Because $\alpha_{t, R}(\lambda) \rightarrow 1$ as $R \rightarrow \infty$, the first term on the right-hand side of (22) tends to zero uniformly as $R$ tends to infinity. Since $0 \leq \alpha_{t, R}\left(\lambda_{n}\right) \leq 1$ for $n>N$, the absolute value of the second term on the right-hand side of (22) is bounded by

$$
\sum_{n=N+1}^{\infty}\left(1-\alpha_{t, R}\left(\lambda_{n}\right)\right)\left|a_{n}\right| \sup \left|\psi_{n}\right|
$$

independently of $x$. This expression tends to zero by Dominated Convergence, in light of (21). This establishes the desired uniform pointwise convergence result.

\section{THE ISOMETRY FoRMULA}

\subsection{Strategy for the isometry formula}

We continue to assume that $G / K$ is a noncompact symmetric space of the complex type (i.e., $G$ is complex) and that $X=\Gamma \backslash G / K$ is a compact quotient of $G / K$ of the sort described in Section 2 .

To obtain the isometry formula for $X$, we will write (heuristically)

$$
\begin{aligned}
\langle f, f\rangle_{L^{2}(X)} & =\left\langle e^{-t \Delta / 2} e^{t \Delta / 2} f, e^{-t \Delta / 2} e^{t \Delta / 2} f\right\rangle_{L^{2}(X)} \\
& =\left\langle F, e^{-t \Delta} F\right\rangle_{L^{2}(X)} .
\end{aligned}
$$

Note that to compute $e^{-t \Delta} F$ (where $F=e^{t \Delta / 2} f$ ), we want to apply the backward heat operator for time $2 t$, rather than just for time $t$. Reasoning as in the previous section, we may compute this backward heat operator by the following integral

$$
e^{-t \Delta} F=\lim _{R \rightarrow \infty} e^{t|\rho|^{2}} \int_{T_{x}^{2 R}(X)} F\left(\exp _{x} i Y\right) j_{x}^{\mathrm{c}}(Y)^{1 / 2} \frac{e^{-|Y|^{2} / 4 t}}{(4 \pi t)^{d / 2}} d Y .
$$

Here it is convenient to integrate over a ball of radius $2 R$ rather than radius $R$, simply to avoid a factor of 2 later on. Heuristically, then, we should have

$$
\langle f, f\rangle_{L^{2}(X)}=\lim _{R \rightarrow \infty} e^{t|\rho|^{2}} \int_{X} \overline{F(x)} \int_{T_{x}^{2 R}(X)} F\left(\exp _{x} i Y\right) j_{x}^{\mathrm{c}}(Y)^{1 / 2} \frac{e^{-|Y|^{2} / 4 t}}{(4 \pi t)^{d / 2}} d Y d x
$$

The crucial next step is a "holomorphic change of variable," which will show that (at least for small $R$ )

$$
\begin{aligned}
& e^{t|\rho|^{2}} \int_{X} \overline{F(x)} \int_{T_{x}^{2 R}(X)} F\left(\exp _{x} i Y\right) j^{\mathrm{c}}(Y)^{1 / 2} \frac{e^{-|Y|^{2} / 4 t}}{(4 \pi t)^{d / 2}} d Y \\
= & e^{t|\rho|^{2}} \int_{X} \int_{T_{x}^{2 R}(X)} \overline{F\left(\exp _{x} i Y / 2\right)} F\left(\exp _{x} i Y / 2\right) j^{\mathrm{c}}(Y)^{1 / 2} \frac{e^{-|Y|^{2} / 4 t}}{(4 \pi t)^{d / 2}} d Y d x .
\end{aligned}
$$


(Compare Lemma 9 of [19] and Section 4 of [52] in the compact case.) Making the change of variable $Y \rightarrow 2 Y$ (for sake of convenience) we obtain a proposal for the form that the isometry theorem should take:

$$
\langle f, f\rangle_{L^{2}(X)}=\lim _{R \rightarrow \infty} e^{t|\rho|^{2}} \int_{X} \int_{T_{x}^{R}(X)}\left|F\left(\exp _{x} i Y\right)\right|^{2} j_{x}^{\mathrm{c}}(2 Y) \frac{e^{-|Y|^{2} / t}}{(\pi t)^{d / 2}} d Y d x
$$

This formula is precisely analogous to what we obtained [30, Thm. 7] for globally symmetric spaces of the complex type, and is as parallel as possible to the isometry formula for the dual compact group case (Eq. (1)).

\subsection{The holomorphic change of variable and the partial isometry formula}

To proceed rigorously, we consider, for a fixed small value of $R$, the integral on the right-hand side of (24). We evaluate this integral by a simple modification of Theorem 3. Then we will establish the holomorphic change of variable in (25), which will lead to a rigorous version of the isometry formula.

Theorem 7. Let $R_{0}$ be as in Proposition 2. For all $R<R_{0} / 2$, let $B_{t, R}$ be the operator defined by

$$
B_{t, R}(f)(x)=e^{t|\rho|^{2}} \int_{T_{x}^{2 R}(X)} F\left(\exp _{x} i Y\right) j_{x}^{\mathrm{c}}(Y)^{1 / 2} \frac{e^{-|Y|^{2} / 4 t}}{(4 \pi t)^{d / 2}} d Y,
$$

where $F:=e^{t \Delta / 2} f$. Then $B_{t, R}$ is a bounded operator on $L^{2}(X)$ and is given by

$$
B_{t, R}=\beta_{t, R}(-\Delta),
$$

where $\beta_{t, R}:[0, \infty) \rightarrow \mathbb{R}$ is given by

$$
\beta_{t, R}(\lambda)=e^{-t \lambda / 2} e^{t|\rho|^{2}} \int_{\substack{Y \in \mathbb{R}^{d} \\|Y| \leq 2 R}} \exp \left(\sqrt{\lambda-|\rho|^{2}} y_{1}\right) \frac{e^{-|Y|^{2} / 4 t}}{(4 \pi t)^{d / 2}} d Y .
$$

Here $\sqrt{\lambda-|\rho|^{2}}$ is either of the two square roots of $\lambda-|\rho|^{2}$.

The proof of this is the same as the proof of Theorem 3 , except that $t$ is replaced by $2 t$ and $R$ by $2 R$ in the appropriate places. Note that if we let $R$ tend to infinity in the definition of $\beta_{t, R}$, we obtain an easily evaluated Gaussian integral, which gives

$$
\lim _{R \rightarrow \infty} \beta_{t, R}(\lambda)=e^{t \lambda / 2} .
$$

This reflects the idea that $B_{t, R} f$ is an approximation to the backward heat operator at time $2 t$, applied to the function $F:=e^{t \Delta / 2} f$. Note that although the right-hand 
side of (27) is an unbounded function of $\lambda, \beta_{t, R}$ is a bounded function of $\lambda$ for each fixed finite value of $R$, as is easily seen from (26).

As in Section 2, for each $x$ in $X=\Gamma \backslash G / K$, we can pick $\tilde{x} \in G / K$ mapping to $x$ under quotienting by the action of $\Gamma$. We can then pick $g \in G$ with $g \cdot x_{0}=\tilde{x}$, where $x_{0}$ is the identity coset in $G / K$. Having made these choices, we get an identification of $T_{x}(X)$ with $T_{x_{0}}(G / K)=\mathfrak{p}$. Two identifications of this sort differ only by the action of $K$ on $\mathfrak{p}$. This means that if we have some $K$-invariant function on $\mathfrak{p}$, we can transfer this function in an unambiguous way to each $T_{x}(X)$.

Theorem 8. ([Holomorphic Change of Variable]). Let $F_{1}$ and $F_{2}$ be holomorphic functions on $T^{R_{0}}(X)$ for some $R_{0}>0$. Let $\alpha$ be a bounded, measurable, $K$-invariant function on $\mathfrak{p}^{R_{0}}$. Then for all $R<R_{0} / 2$ we have

$$
\begin{aligned}
& \int_{X} \overline{F_{1}(x)} \int_{T_{x}^{2 R}(X)} F_{2}\left(\exp _{x} i Y\right) \alpha(Y) d Y d x \\
= & \int_{X} \int_{T_{x}^{2 R}(X)} \overline{F_{1}\left(\exp _{x}(i Y / 2)\right)} F_{2}\left(\exp _{x}(i Y / 2)\right) \alpha(Y) d Y d x .
\end{aligned}
$$

Note that although the right-hand side of (28) is defined for all $R<R_{0}$, the left-hand side is defined only for $R<R_{0} / 2$. Once this result is established, we will apply Theorem 7 and the holomorphic change of variable with $\alpha$ given by

$$
\alpha(Y)=\nu_{2 t}^{\mathrm{c}}(Y) j^{\mathrm{c}}(Y)=e^{t|\rho|^{2}} j^{\mathrm{c}}(Y)^{1 / 2} \frac{e^{-|Y|^{2} / 4 t}}{(4 \pi t)^{d / 2}} .
$$

After making the change of variable $Y \rightarrow 2 Y$, for convenience, we will obtain the following result, which is the main result of this subsection.

Theorem 9. ([Partial Isometry Formula]). Given $f_{1}, f_{2} \in L^{2}(X)$, let $F_{1}=$ $e^{t \Delta / 2} f_{1}$ and $F_{2}=e^{t \Delta / 2} f_{2}$. Let $R_{0}$ be as in Proposition 2. Then for all $R<R_{0}$,

$$
\begin{aligned}
& e^{t|\rho|^{2}} \int_{X} \int_{T_{x}^{R}(X)} \overline{F_{1}\left(\exp _{x}(i Y)\right)} F_{2}\left(\exp _{x}(i Y)\right) j^{\mathrm{c}}(2 Y)^{1 / 2} \frac{e^{-|Y|^{2} / t}}{(\pi t)^{d / 2}} d Y d x \\
= & \left\langle f_{1}, e^{t \Delta / 2} \beta_{t, R}(-\Delta) f_{2}\right\rangle_{L^{2}(X)},
\end{aligned}
$$

where $\beta_{t, R}$ is the function defined in (26).

The analogous result on the globally symmetric space $G / K$ ( $G$ complex) was obtained in [30]; see Theorem 6 and Equations (38) and (39). We will prove (30) directly from the holomorphic change of variable for $R<R_{0} / 2$ and then extend the result to $R<R_{0}$ by analytic continuation.

From the formula (8), we see that $j^{\mathrm{c}}(2 Y)$ is positive for all sufficiently small $Y$. (Actually, it is not hard to show that $j^{\mathrm{c}}(2 Y)$ is positive on the maximal domain 
in $T(X)$ on which the adapted complex structure exists, but we do not require this result.) This means that the left-hand side of (30) is strictly positive whenever $f_{1}=f_{2}=f$, where $f$ is nonzero. If follows that $\beta_{t, R}(-\Delta)$ is a strictly positive operator, for all sufficiently small $R$, something that can also be obtained from the formula for $\beta_{t, R}$. However, because the spectrum of $-\Delta$ contains points in the interval $\left[0,|\rho|^{2}\right)$, it is not clear whether positivity holds for all $R$.

Note that the operator $B_{t, R}$ in Theorem 7 incorporates the $e^{t \Delta / 2}$ applied to $f_{1}$, but not the $e^{t \Delta / 2}$ applied to $f_{2}$. This, along with the self-adjointness of $e^{t \Delta / 2}$, accounts for the expression on the right-hand side of (30).

To understand what is going on in Theorem 8 , it is helpful to consider the following prototype calculation on the (Euclidean) symmetric space $S^{1}=\mathbb{R} / \mathbb{Z}$. Then $T(\mathbb{R} / \mathbb{Z})$, with the adapted complex structure, is identified with $\mathbb{C} / \mathbb{Z}$ in such a way that $\exp _{x}(i y)=x+i y$. Suppose $F_{1}$ and $F_{2}$ are holomorphic functions on a strip in $\mathbb{R} / \mathbb{Z}$. Let $\tilde{F}_{1}$ be the holomorphic function whose restriction to $\mathbb{R} / \mathbb{Z}$ is $\overline{F_{1}}$; equivalently, $\tilde{F}_{1}(x+i y)=\overline{F_{1}(x-i y)}$. Then using Fubini and a change of variable we have

$$
\begin{aligned}
& \int_{\mathbb{R} / \mathbb{Z}} \overline{F_{1}(x)} \int_{-2 R}^{2 R} F_{2}(x+i y) \alpha(y) d y d x \\
= & \int_{-2 R}^{2 R} \int_{\mathbb{R} / \mathbb{Z}} \tilde{F}_{1}(x-a) F_{2}(x-a+i y) d x \alpha(y) d y
\end{aligned}
$$

for any $a \in \mathbb{R}$. Since $\tilde{F}_{1}$ and $F_{2}$ are holomorphic, this equality remains valid for $a$ in a strip in $\mathbb{C}$. Taking $a=i y / 2$ and using Fubini again gives

$$
\begin{aligned}
& \int_{\mathbb{R} / \mathbb{Z}} \overline{F_{1}(x)} \int_{-2 R}^{2 R} F_{2}(x+i y) \alpha(y) d y d x \\
= & \int_{\mathbb{R} / \mathbb{Z}} \int_{-2 R}^{2 R} \overline{F_{1}(x+i y / 2)} F_{2}(x+i y / 2) \alpha(y) d y d x .
\end{aligned}
$$

This is just the analog of Theorem 8 for $\mathbb{R} / \mathbb{Z}$ and the method of proof (the "change of variable" $x \rightarrow x-i y / 2)$ motivates the terminology "holomorphic change of variable".

The remainder of this subsection is devoted to the proof of Theorem 8. The first step is to express the two sides of (28) in terms of "double orbital integrals," with the integration being over $\Gamma \backslash G$ with respect to the natural right- $G$-invariant measure. Then a "holomorphic change of variable" in the double orbital integrals, similar to that in the previous paragraph, will show that the integrals are equal.

Let $\pi: G / K \rightarrow X=\Gamma \backslash G / K$ be the quotient map and let $x_{0}$ denote the identity coset in $G / K$. For each $x \in X$, we choose $g_{x} \in G$ so that $\pi\left(g_{x} \cdot x_{0}\right)=x$. We arrange for the $g_{x}$ 's to depend measurably on $x$, and we will make one other 
technical restriction on the choice of $g_{x}$ later. We then identify each $T_{x}(X)$ with $T_{g_{x} \cdot x_{0}}(G / K)$ by means of $\pi_{*}^{-1}$ and then with $\mathfrak{p}=T_{x_{0}}(G / K)$ by the action of $g_{x}^{-1}$. This identification of $T_{x}(X)$ with $\mathfrak{p}$ is of the same sort as we have been using all along in this paper, but we now have one particular such identification for each $x$.

We have now measurably identified $T(X)$ with $X \times \mathfrak{p}$. We use this identification on both sides of the desired equality (28), along with generalized polar coordinates on $\mathfrak{p}$ with respect to the adjoint action of $K$. Recall (from (6)) that $\exp _{x}(i Y)$ is simply another way of writing the point $(x, Y) \in T(X)$. Let us now switch back to the $(x, Y)$ notation. Then, using our identification of $T(X)$ with $X \times \mathfrak{p}$ and generalized polar coordinates on $\mathfrak{p}$, the desired equality (28) is equivalent to

$$
\begin{aligned}
& \int_{\mathfrak{a}_{2 R}^{+}} \int_{X} \int_{K} \overline{F_{1}(x)} F_{2}\left(\left(x, \operatorname{Ad}_{k} Y\right)\right) d k d x \alpha(Y) \mu(Y) d Y \\
= & \int_{\mathfrak{a}_{2 R}^{+}} \int_{X} \int_{K} \overline{F_{1}\left(\left(x, \operatorname{Ad}_{k} Y / 2\right)\right)} F_{2}\left(\left(x, \operatorname{Ad}_{k} Y / 2\right)\right) d k d x \alpha(Y) \mu(Y) d Y .
\end{aligned}
$$

Here, $d x$ denotes the Riemannian volume measure on $X, \mu$ is the density that appears in the generalized polar coordinates formula (e.g., [33, Thm. I.5.17]), and $\mathfrak{a}_{2 R}^{+}$is the set of vectors in $\mathfrak{a}^{+}$with norm less than $2 R$.

Clearly, for (31) to hold, it is sufficient to verify that

$$
\begin{aligned}
& \int_{X} \int_{K} \overline{F_{1}(x)} F_{2}\left(\left(x, \operatorname{Ad}_{k} Y\right)\right) d k d x \\
= & \int_{X} \int_{K} \overline{F_{1}\left(\left(x, \operatorname{Ad}_{k} Y / 2\right)\right)} F_{2}\left(\left(x, \operatorname{Ad}_{k} Y / 2\right)\right) d k d x
\end{aligned}
$$

for all $Y \in \mathfrak{a}_{2 R}^{+}$.

Our goal now is to show that both integrals in (32) can be written as "double orbital integrals." Let $\tilde{F}_{1}$ be the function on $T^{R_{0}}(X)$ given by $\tilde{F}_{1}(x, Y)=\overline{F_{1}(x,-Y)}$ (or, by $\left.(6), \tilde{F}_{1}\left(\exp _{x} i Y\right)=\overline{F_{1}\left(\exp _{x}(-i Y)\right)}\right)$. Since the map $(x, Y) \rightarrow(x,-Y)$ is antiholomorphic [16, p. 568], $\tilde{F}_{1}$ is holomorphic. Now, it is known that $T^{R}(G / K)$ has its own adapted complex structure for all sufficiently small $R$ (see [30] and the references therein). Furthermore, the map $\pi_{*}: T^{R}(G / K) \rightarrow T^{R}(\Gamma \backslash G / K)$ is easily seen to be holomorphic. Thus, we can define holomorphic functions $\Phi_{1}$ and $\Phi_{2}$ on $T^{R}(G / K)$ by

$$
\begin{aligned}
& \Phi_{1}=\tilde{F}_{1} \circ \pi_{*} \\
& \Phi_{2}=F_{2} \circ \pi_{*} .
\end{aligned}
$$

By construction, these functions satisfy $\Phi_{j}(\gamma \cdot a)$ for all $\gamma \in \Gamma$ and $a \in T^{R}(G / K)$, where $\gamma \cdot a$ refers to the action of $\Gamma$ on $T^{R}(G / K)$ induced from the action of $\Gamma$ on $G / K$.

We now consider "double orbital integral," namely, integrals of the form 


$$
\int_{\Gamma \backslash G} \Phi_{1}(g \cdot a) \Phi_{2}(g \cdot b) d g,
$$

where $d g$ is an appropriately normalized version of the right- $G$-invariant measure on $\Gamma \backslash G$ and $a$ and $b$ are fixed points in $T^{R}(G / K)$. Observe that although $g \cdot a$ and $g \cdot b$ are defined for $g \in G$ (not $\Gamma \backslash G$ ), the invariance property of the $\Phi_{j}$ 's means that the integrand in (33) descends to a function on $\Gamma \backslash G$.

Lemma 10. The left-hand side of (32) is an integral of the form (33) with $a=x_{0}$ and $b=\left(x_{0}, Y\right)$. The right-hand side of (32) is an integral of the form (33) with $a=\left(x_{0},-Y / 2\right)$ and $b=\left(x_{0}, Y / 2\right)$.

Proof. Let $E$ denote the following set in $G$ :

$$
E=\left\{g_{x} k \in G \mid x \in X, k \in K\right\} .
$$

We assume that the mapping $x \rightarrow g_{x}$ is chosen in such a way that $E$ is a measurable subset of $G$.

Now consider the map from $X \times K$ to $\Gamma \backslash G$ given by $(x, k) \rightarrow \Gamma g_{x} k$. This map is measurable because the map $x \rightarrow g_{x}$ was chosen to be measurable. Given $\Gamma g \in$ $\Gamma \backslash G$, let $x$ be the point $\Gamma g K \in \Gamma \backslash G / K=X$ and then consider $g_{x} \in G$, which has the property that $\Gamma g_{x} K=x$. Then there exists $k \in K$ with $\Gamma g_{x} k=\Gamma g$. To see that $k$ is unique, suppose $\gamma_{1} g_{x} k_{1}=\gamma_{2} g_{x} k_{2}$ for some $\gamma_{1}, \gamma_{2} \in \Gamma$ and $k_{1}, k_{2} \in K$. Then because $\Gamma$ acts freely on $G / K$, we must have $\gamma_{1}=\gamma_{2}$ and therefore $k_{1}=k_{2}$.

This argument shows that the map $(x, k) \rightarrow \Gamma g_{x} k$ is a bijection of $X \times K$ onto $\Gamma \backslash G$. We may therefore identify $\Gamma \backslash G$ with the set $E \subset G$ defined above. Consider on $X \times K$ the product of the Riemannian volume measure on $X$ and the normalized Haar measure on $K$. The push-forward of this measure to $E$ is easily seen to be the restriction to $E$ of a (bi-invariant) Haar measure on $G$. (Specifically, arguing as in the proof of Proposition 4 in [30], the push-forward measure is the restriction to $E$ of a left Haar measure on $G$, which is also right invariant since $G$ is unimodular.) It is then easy to see that if we identify $E$ with $\Gamma \backslash G$, the resulting measure on $\Gamma \backslash G$ is invariant under the right action of $G$.

All of this is to say that if we write points in $\Gamma \backslash G$ as $\Gamma g_{x} k$, with $x \in X$ and $k \in K$, then the measure $d g$ on $\Gamma \backslash G$ decomposes as $d x d k$. Meanwhile, in light of the identifications we are making,

$$
\pi_{*}\left[\left(g_{x} k\right) \cdot\left(x_{0}, Z\right)\right]=\left(x, \operatorname{Ad}_{k} Z\right), \quad Z \in \mathfrak{p} .
$$

The lemma then follows by plugging in $Z=0, Z=Y, Z=-Y / 2$, and $Z=Y / 2$.

\section{Lemma 11.}

(1) Integrals of the form (33) satisfy

$$
\int_{\Gamma \backslash G} \Phi_{1}(g \cdot(h \cdot a)) \Phi_{2}(g \cdot(h \cdot b)) d g=\int_{\Gamma \backslash G} \Phi_{1}(g \cdot a) \Phi_{2}(g \cdot b) d g
$$


for all $h \in G$ and $a, b \in T^{R}(G / K)$.

(2) The integral in (33) depends holomorphically on $a$ and $b$ (with $F_{1}$ and $F_{2}$ and hence $\Phi_{1}$ and $\Phi_{2}$ fixed).

Proof. The first point follows from the associativity property of the action and the right- $G$-invariance of the measure on $\Gamma \backslash G$. The second point follows from Morera's Theorem.

We are now ready to give the proof of the holomorphic change of variable.

Proof of Theorem 8. It suffices to prove (32), which, by Lemma 10 amounts to showing that the (double) orbital integral with $a=x_{0}$ and $b=\left(x_{0}, Y\right)$ is the same as the orbital integral with $a=\left(x_{0},-Y / 2\right)$ and $b=\left(x_{0}, Y / 2\right)$. The idea is to use invariance of the orbital integral under $(a, b) \rightarrow\left(e^{t Y} \cdot a, e^{t Y} \cdot b\right)$ and then plug in $t=-i / 2$, using Lemma 11 .

There is nothing to prove in (32) if $Y=0$. If $Y \neq 0$, let $r=|Y|$ (with $r<R_{0}$ ) and let $X=Y / r$ be the associated unit vector. Let $\gamma$ be the corresponding unit-speed geodesic in $G / K$, namely, $\gamma(t)=e^{t X} \cdot x_{0}$. Then consider the map $\tau: S_{R} \rightarrow T(G / K)$ given by

$$
\tau(u+i v)=(\gamma(u), v \dot{\gamma}(u))
$$

where $S_{R} \subset \mathbb{C}$ is the strip defined in (5). According to the definition of the adapted complex structure on $T(G / K)$, the map $\tau$ is holomorphic. Note that

$$
\frac{d}{d t} e^{t X} \cdot x_{0}=\left.\frac{d}{d \varepsilon} e^{(t+\varepsilon) X} \cdot x_{0}\right|_{\varepsilon=0}=\left.\frac{d}{d \varepsilon} e^{t X} \cdot\left(e^{\varepsilon X} \cdot x_{0}\right)\right|_{\varepsilon=0}=e^{t X} \cdot\left(x_{0}, X\right),
$$

where in the last expression we have the induced action of $e^{t X}$ on $T(G / K)$. It follows that

$$
\tau(u+i v)=e^{u X} \cdot\left(x_{0}, v X\right) .
$$

By the first part of Lemma 11, the orbital integral with $a=x_{0}$ and $b=\left(x_{0}, r X\right)$ is the same as the orbital integral with $a=e^{t X} \cdot x$ and $b=e^{t X} \cdot\left(x_{0}, r X\right)$, for all $t \in \mathbb{R}$. That is, the orbital integral associated to $a=\tau(t)$ and $b=\tau(t+i r)$ is independent of $t$ for $t \in \mathbb{R}$. Since $\tau$ is holomorphic, the second part of Lemma 11 tells us the same result for all $t \in \mathbb{C}$ such that both $t$ and $t+i r$ belong to the strip $S_{R}$. Equating the orbital integral with $(a, b)=(\tau(0), \tau(i r))$ (i.e., $\left.t=0\right)$ to the one with $(a, b)=(\tau(-i r / 2), \tau(i r / 2))$ (i.e., $t=-i r / 2)$ gives the desired result.

Proof of Theorem 9. For $R<R_{0} / 2$, this result follows from applying the holomorphic change of variable with $\alpha$ as in (29) (and then making the cosmetic change of variable $Y \rightarrow 2 Y$ ). The result will hold for all $R<R_{0}$, provided we can show that both sides of (30) are real-analytic in $R$. The analyticity of the right-hand side of (30) is established as part of the proof of Theorem 13 in Section 4.3. The analyticity of the left-hand side is established in the following lemma. 
Lemma 12. Let $R_{1}$ be any positive real number such that the adapted complex structure exists on $T^{R_{1}}(X)$. Let $F$ be a holomorphic function on $T^{R_{1}}(X)$ and let $\alpha$ be a real-analytic, Ad-K-invariant function on $\mathfrak{p}^{2 R_{1}}$. Then the function $G_{F}$ defined by

$$
G_{F}(R)=\int_{X} \int_{T_{x}^{2 R}(X)} \overline{F_{1}\left(\exp _{x}(i Y / 2)\right)} F_{2}\left(\exp _{x}(i Y / 2)\right) \alpha(Y) d Y d x
$$

is real-analytic on the interval $\left(0, R_{1}\right)$.

Proof. We measurably identify $T(X)$ with $X \times \mathfrak{p}$ and then decompose $G_{F}(R)$ as in the right-hand side of (31). We let $\Phi_{1}=\tilde{F}_{1} \circ \pi_{*}$ and we let $\Phi_{2}=F_{2} \circ \pi_{*}$, as before. In light of Lemma 11, we can then write our function as

$$
\begin{aligned}
& G_{F}(R) \\
= & \int_{\mathfrak{a}_{2 R}^{+}} \int_{\Gamma \backslash G} \Phi_{1}\left(g \cdot\left(x_{0}, Y / 2\right)\right) \Phi_{2}\left(g \cdot\left(x_{0},-Y / 2\right)\right) d g \alpha(Y) \mu(Y) d Y \\
= & \int_{\mathfrak{a}_{1}^{+}} \int_{\Gamma \backslash G} \Phi_{1}\left(g \cdot\left(x_{0}, R Z\right)\right) \Phi_{2}\left(g \cdot\left(x_{0},-R Z\right)\right) d g(2 R)^{d} \alpha(2 R Z) \mu(2 R Z) d Z .
\end{aligned}
$$

Let $\tau_{Z}$ be the map from the strip $S_{R_{1}} \subset \mathbb{C}$ into $T^{R_{1}}(G / K)$ given by $\tau_{Z}(u+i v)=$ $e^{u Z} \cdot\left(x_{0}, v Z\right)$. As in the proof of Theorem 8 , this map is holomorphic. (There it was assumed that $Z$ was a unit vector, but by scaling the statement is true for any $Z$ with $|Z|<1$.) Note that $\left(x_{0}, R Z\right)=\tau_{Z}(i R)$ and $\left(x_{0},-R Z\right)=\tau_{Z}(-i R)$. Then given any $R \in\left(0, R_{1}\right)$, we claim that $G_{F}$ has a holomorphic extension to a small neighborhood of $R$ in $\mathbb{C}$, given by

$$
\begin{aligned}
& G_{F}(S) \\
= & \int_{\mathfrak{a}_{1}^{+}} \int_{\Gamma \backslash G} \Phi_{1}\left(g \cdot \tau_{Z}(i S)\right) \Phi_{2}\left(g \cdot \tau_{Z}(-i S)\right) d g(2 S)^{d} \alpha(2 S Z) \mu(2 S Z) d Z .
\end{aligned}
$$

This follows from Point 2 of Lemma 11 and the analyticity of $\alpha$ (assumed) and $\mu$ (it is a polynomial).

To be a bit more precise, $\alpha$ is assume to be real-analytic on $\mathfrak{p}^{2 R_{1}}$. Thus, for each $R<R_{1}, \alpha$ has a holomorphic extension to a neighborhood $U$ in $\mathfrak{p}_{\mathbb{C}}$ of the closed ball of radius $2 R$ in $\mathfrak{p}$. Then for all $S$ in a neighborhood of $R, 2 S Z$ will belong to $U$ for all $Z \in \mathfrak{p}$ with $|Z| \leq 1$. For $S$ in a slightly smaller neighborhood of $R, \alpha(2 S Z)$ will be bounded uniformly in $Z$ with $|Z| \leq 1$. The integrand in (34) is thus holomorphic in $S$ and bounded uniformly in $g$ and $Z$, from which it follows that the integral is holomorphic in $S$.

The existence of this holomorphic extension establishes the real-analyticity of $G_{F}$. 


\subsection{The global isometry formula}

Theorem 13. ([Global Isometry Formula]). Given $f \in L^{2}(X)$ and let $F=$ $e^{t \Delta / 2} f$. Let $R_{0}$ be as in Proposition 2. Then the quantity

$$
G_{F}(R):=e^{t|\rho|^{2}} \int_{X} \int_{T_{x}^{R}(X)}\left|F\left(\exp _{x}(i Y)\right)\right|^{2} j_{x}^{\mathrm{c}}(2 Y)^{1 / 2} \frac{e^{-|Y|^{2} / t}}{(\pi t)^{d / 2}} d Y d x,
$$

initially defined for $R \in\left(0, R_{0}\right)$, has a real-analytic extension to $R \in(0, \infty)$. Furthermore, this real-analytic extension, also denoted $G_{F}$, satisfies

$$
\lim _{R \rightarrow \infty} G_{R}(R)=\|f\|_{L^{2}(X)}^{2} .
$$

Thus, we may write, informally,

$$
\begin{aligned}
& \|f\|_{L^{2}(X)}^{2} \\
& =“ \lim _{R \rightarrow \infty} " e^{t|\rho|^{2}} \int_{X} \int_{T_{x}^{R}(X)}\left|F\left(\exp _{x}(i Y)\right)\right|^{2} j_{x}^{\mathrm{c}}(2 Y)^{1 / 2} \frac{e^{-|Y|^{2} / t}}{(\pi t)^{d / 2}} d Y d x .
\end{aligned}
$$

This result, as for the inversion formulas, is obtained from the corresponding result in the dual compact group case (see (1)) by "dualizing" and inserting an analytic continuation with respect to $R$.

In the corresponding theorem for $G / K$ ( $G$ complex) in [30], the proof actually shows that $G_{F}(R)$ is positive and strictly increasing as a function of $R$. In the case of the compact quotient $X=\Gamma \backslash G / K$, however, the presence of spectrum for $-\Delta$ in the interval $\left[0,|\rho|^{2}\right)$ means that $G_{F}(R)$ is not necessarily monotone in $R$, once $R>R_{0}$. (See (36) below.)

Proof. Putting $f_{1}=f_{2}=f$ in the partial isometry formula (Theorem 9), we have (with $F=e^{t \Delta / 2} f$ as usual)

$$
G_{F}(R)=\left\langle f, e^{t \Delta / 2} \beta_{t, R}(-\Delta) f\right\rangle_{L^{2}(X)} .
$$

The point is now that the definition of $\beta_{t, R}$ makes sense for any $R>0$. Thus, the right-hand side of (35) makes sense for all $R>0$, even though the left-hand side is defined only for small $R$. Equation (27) then suggests that the right-hand side of (35) should tend to $\langle f, f\rangle_{L^{2}(X)}$ as $R$ tends to infinity.

To proceed rigorously, we choose an orthonormal basis $\left\{\psi_{n}\right\}$ for $L^{2}(X)$ consisting of eigenvectors of $-\Delta$ with eigenvalues $\lambda_{n}$. Then if $f=\sum a_{n} \psi_{n}$, we have, for any $R>0$, 


$$
\begin{aligned}
& \left\langle f, e^{t \Delta / 2} \beta_{t, R}(-\Delta) f\right\rangle_{L^{2}(X)} \\
= & \sum_{n=1}^{\infty}\left|a_{n}\right|^{2} e^{-t \lambda_{n} t / 2} \beta_{t, R}\left(\lambda_{n}\right) \\
= & \sum_{n=1}^{\infty}\left|a_{n}\right|^{2} e^{-t \lambda_{n}} e^{t|\rho|^{2}} \int_{|Y| \leq R} \exp \left(\sqrt{\lambda_{n}-|\rho|^{2}} y_{1}\right) \frac{e^{-|Y|^{2} / 4 t}}{(4 \pi t)^{d / 2}} d Y .
\end{aligned}
$$

The same argument as in the proof of Theorem 4 shows that Fubini's Theorem applies, so that we obtain

$$
\begin{aligned}
& \left\langle f, e^{t \Delta / 2} \beta_{t, R}(-\Delta) f\right\rangle_{L^{2}(X)} \\
= & e^{c t} \int_{|Y| \leq R}\left[\sum_{n=1}^{\infty}\left|a_{n}\right|^{2} e^{-t \lambda_{n}} \exp \left(\sqrt{\lambda_{n}-|\rho|^{2}} y_{1}\right)\right] \frac{e^{-|Y|^{2} / 4 t}}{(4 \pi t)^{d / 2}} d Y .
\end{aligned}
$$

Arguing, again, as in the proof of Theorem 4, we can see that the expression in square brackets has an entire holomorphic extension to $y_{1} \in \mathbb{C}$ (given by the same expression) and that the whole right-hand side of (37) is real-analytic as a function of $R$, for all $R \in(0, \infty)$.

We have established, then, that the right-hand side of (35) is a real-analytic function of $R$ for $R \in(0, \infty)$. This, along with Lemma 12, shows that (35) holds for all $R<R_{0}$. (This was initially established, using the holomorphic change of variable, only for $R<R_{0} / 2$.) Thus, the right-hand side of (35) is the desired real-analytic extension of $G_{F}$. To evaluate the limit as $R$ tends to infinity of this expression, we use our orthonormal basis $\left\{\psi_{n}\right\}$ and we find $N$ so that $\lambda_{n} \geq|\rho|^{2}$ for $n>N$. Then

$$
\begin{aligned}
& \left\langle f, e^{t \Delta / 2} \beta_{t, R}(-\Delta) f\right\rangle_{L^{2}(X)} \\
= & \sum_{n=1}^{N}\left|a_{n}\right|^{2} e^{-t \lambda_{n} / 2} \beta_{t, R}\left(\lambda_{n}\right)+\sum_{n=N+1}^{\infty}\left|a_{n}\right|^{2} e^{-t \lambda_{n} / 2} \beta_{t, R}\left(\lambda_{n}\right) .
\end{aligned}
$$

Note that (by 27) we have

$$
\lim _{R \rightarrow \infty} e^{-t \lambda / 2} \beta_{t, R}(\lambda)=1
$$

for all $\lambda \geq 0$. For $n>N, \beta_{t, R}\left(\lambda_{n}\right)$ is positive and increasing with $R$; thus, by Monotone Convergence, we can put the limit as $R$ tends to infinity inside the infinite sum in (38). This shows that

$$
\lim _{R \rightarrow \infty}\left\langle f, e^{t \Delta / 2} \beta_{t, R}(-\Delta) f\right\rangle_{L^{2}(X)}=\sum_{n=1}^{\infty}\left|a_{n}\right|^{2}=\|f\|_{L^{2}(X)}^{2} .
$$

This, in light of (35), is what we want to prove. 


\subsection{The surjectivity theorem}

We now show, roughly, that if $F$ is any holomorphic function for which the right-hand side of the global isometry formula makes sense and is finite, then $F$ is the analytic continuation of a function of the form $e^{t \Delta / 2} f$, with $f \in L^{2}(X)$.

Theorem 14. Suppose $F$ is a holomorphic function on $T^{R_{1}}(X)$, for some $R_{1}>0$ such that the adapted complex structure exists on $T^{R_{1}}(X)$. Let $G_{F}$ be the function defined by

$$
G_{F}(R)=e^{t|\rho|^{2}} \int_{X} \int_{T_{x}^{R}(X)}\left|F\left(\exp _{x}(i Y)\right)\right|^{2} j_{x}^{\mathrm{c}}(2 Y)^{1 / 2} \frac{e^{-|Y|^{2} / t}}{(\pi t)^{d / 2}} d Y d x,
$$

for $R<R_{1}$. Suppose that $G_{F}$ has a real-analytic extension (also denoted $G_{F}$ ) from $\left(0, R_{1}\right)$ to $(0, \infty)$ and that

$$
\lim _{R \rightarrow \infty} G_{F}(R)
$$

exists and is finite. Then there exists a unique $f \in L^{2}(X)$ for which $\left.F\right|_{X}=e^{t \Delta / 2} f$.

Choose $R_{0}$ so as in Proposition 2 and then choose $R_{2} \leq R_{0}$ so that the function $j^{\mathrm{c}}(2 Y)$ is positive on $T^{R_{2}}(X)$. For any $R<R_{2}$, let $\mathcal{H} L^{2}\left(T^{R}(X)\right)_{t}$ denote the space of holomorphic functions $F$ on $T^{R}(X)$ for which

$$
e^{t|\rho|^{2}} \int_{X} \int_{T_{x}^{R}(X)}\left|F\left(\exp _{x}(i Y)\right)\right|^{2} j_{x}^{\mathrm{c}}(2 Y)^{1 / 2} \frac{e^{-|Y|^{2} / t}}{(\pi t)^{d / 2}} d Y d x<\infty,
$$

with the obvious associated inner product. A standard argument shows that $\mathcal{H} L^{2}$ $\left(T^{R}(X)\right)_{t}$ is a closed subspace of the associated $L^{2}$ space, and hence a Hilbert space. Note that if $\psi \in L^{2}(X)$ is an eigenvector for $-\Delta$ with eigenvalue $\lambda$, then $\psi=e^{t \Delta / 2}\left(e^{\lambda t / 2} \psi\right)$, so that by Proposition $2, \psi$ has an analytic continuation to $T^{R_{0}}(X)$. This analytic continuation is bounded on each $T^{R}(X)$ for $R<R_{0}$ and hence belongs to $\mathcal{H} L^{2}\left(T^{R}(X)\right)_{t}$.

Lemma 15. Let $\left\{\psi_{n}\right\}$ be an orthonormal basis for $L^{2}(X)$ consisting of eigenvectors for $-\Delta$. Let $\psi_{n}$ also denote the analytic continuation of $\psi_{n}$ to $T^{R}(X)$. Then the $\psi_{n}$ 's form an orthogonal basis for $\mathcal{H} L^{2}\left(T^{R}(X)\right)_{t}$, for all $R<R_{2}$ and $t>0$.

Proof. We fix one particular $R<R_{2}$ and $t>0$, and we abbreviate $\mathcal{H} L^{2}\left(T^{R}(X)\right)_{t}$ by $\mathcal{H} L^{2}$. The partial isometry theorem (Theorem 9) tells us that for $n \neq m, \psi_{n}$ and $\psi_{m}$ are orthogonal (but not orthonormal) as elements of $\mathcal{H} L^{2}$.

Suppose now that $F \in \mathcal{H} L^{2}$ and $\left\langle F, \psi_{n}\right\rangle_{\mathcal{H} L^{2}}=0$ for all $n$. By the holomorphic change of variable (Theorem 8), with $\alpha$ as in (29), along with Theorem 7, we have

$$
\left\langle F, \psi_{n}\right\rangle_{\mathcal{H} L^{2}}=\left\langle F, \beta_{t, R}(-\Delta) \psi_{n}\right\rangle_{L^{2}(X)}=\beta_{t, R}\left(\lambda_{n}\right)\left\langle F, \psi_{n}\right\rangle_{L^{2}(X)} .
$$


Here, in the second and third expressions, $F$ denotes the restriction of the holomorphic function $F$ to $X$. Now, as we have already remarked, it follows from the partial isometry theorem (Theorem 9) that $\beta_{t, R}\left(\lambda_{n}\right)$ is strictly positive for all $n$, for all $R<R_{2}$. (See the discussion immediately after the statement of the theorem.) Thus, if $F$ is orthogonal to each $\psi_{n}$ in $\mathcal{H} L^{2}\left(T^{R}(X)\right)_{t}$, then the restriction of $F$ to $L^{2}(X)$ is orthogonal to each $\psi_{n}$. Since the $\psi_{n}$ 's form an orthonormal basis for $L^{2}(X)$, this tells us that the restriction of $F$ to $X$ is zero, from which it follows that $F$ is zero on $T^{R}(X)$, because $X$ is a totally real submanifold of maximal dimension in $T^{R}(X)$.

We now turn to the proof of the surjectivity theorem.

Proof of Theorem 14. Suppose $F$ is as in Theorem 14. The lemma tells us that for $R<\min \left(R_{1}, R_{2}\right)$ we can express $F$ as

$$
F=\sum_{n=1}^{\infty} a_{n} \psi_{n},
$$

with convergence in $\mathcal{H} L^{2}\left(T^{R}(X)\right)_{t}$. By a standard argument, pointwise evaluation is continuous in $\mathcal{H} L^{2}\left(T^{R}(X)\right)_{t}$ with norm a locally bounded function of the point. It follows that the restriction map from that space to $L^{2}(X)$ is a bounded operator. Thus, the same expansion (39) holds also in $L^{2}(X)$. This shows that the coefficients in (39) are independent of $R$ for a fixed holomorphic function $F$.

We apply the partial isometry formula (Theorem 9) with $f_{1}=f_{2}=e^{t \lambda_{n} / 2} \psi_{n}$, so that $F_{1}=F_{2}=\psi_{n}$. This tells us that the norm-squared of $\psi_{n}$ in $\mathcal{H} L^{2}\left(T^{R}(X)\right)_{t}$ is $e^{t \lambda_{n} / 2} \beta_{t, R}\left(\lambda_{n}\right)$ (times the norm-squared of $\psi_{n}$ in $L^{2}(X)$, which is 1 ). We conclude, then, that

$$
\begin{aligned}
G_{F}(R) & =\|F\|_{\mathcal{H} L^{2}\left(T^{R}(X)\right)_{t}}^{2}=\sum_{n=1}^{\infty}\left|a_{n}\right|^{2} e^{t \lambda_{n} / 2} \beta_{t, R}\left(\lambda_{n}\right) \\
& =\sum_{n=1}^{\infty}\left|a_{n}\right|^{2} e^{t|\rho|^{2}} \int_{\substack{Y \in \mathbb{R}^{d} \\
|Y| \leq 2 R}} \exp \left(\sqrt{\lambda_{n}-|\rho|^{2}} y_{1}\right) \frac{e^{-|Y|^{2} / 4 t}}{(4 \pi t)^{d / 2}} d Y,
\end{aligned}
$$

for all $R<\min \left(R_{1}, R_{2}\right)$. We now split off the finite number of terms where $\lambda_{n}<|\rho|^{2}$. Those terms have an analytic continuation in $R$ given by the same expression. For the remaining terms, the argument given in Section 7 of [30] shows that if $G_{F}(R)$ is to have an analytic continuation in $R$ to $(0, \infty)$, it must be given by (40) for all $R$.

We now know that the analytic continuation of $G_{F}$ (which is assumed to exist) is given by (40) for all $R \in(0, \infty)$. To evaluate the limit as $R \rightarrow \infty$ of $G_{F}(R)$, we use Dominated Convergence on the finite number of terms with $\lambda_{n}<|\rho|^{2}$ and we use Monotone Convergence twice on the remaining terms to obtain 


$$
\begin{aligned}
& \lim _{R \rightarrow \infty} G_{F}(R) \\
= & \sum_{n=1}^{\infty}\left|a_{n}\right|^{2} e^{t|\rho|^{2}} \int_{Y \in \mathbb{R}^{d}} \exp \left(\sqrt{\lambda_{n}-|\rho|^{2}} y_{1}\right) \frac{e^{-|Y|^{2} / 4 t}}{(4 \pi t)^{d / 2}} d Y \\
= & \sum_{n=1}^{\infty}\left|a_{n}\right|^{2} e^{t \lambda_{n}} .
\end{aligned}
$$

Since the limit of $G_{F}$ is assumed finite, we conclude that the right-hand side of (41) is finite. We may then define $f=\sum_{n=1}^{\infty} a_{n} e^{t \lambda_{n} / 2} \psi_{n}$. The finiteness of (41) gives convergence of this series in $L^{2}(X)$ and we observe that $\left.F\right|_{X}=$ $\sum_{n=1}^{\infty} a_{n} \psi_{n}=e^{t \Delta / 2} f$. This establishes Theorem 14 .

\section{REFERENCES}

1. D. N. Akhiezer and S. G. Gindikin, On Stein extensions of real symmetric spaces, Math. Ann., 286 (1990), 1-12.

2. V. Bargmann, On a Hilbert space of analytic functions and an associated integral transform, Comm. Pure Appl. Math., 14 (1961), 187-214.

3. D. Burns, S. Halverscheid and R. Hind, The geometry of Grauert tubes and complexification of symmetric spaces, Duke Math. J., 118 (2003), 465-491.

4. M. Davidson, G. Ólafsson and G. Zhang, Laguerre polynomials, restriction principle, and holomorphic representations of SL(2, R), Acta Appl. Math., 71 (2002), 261-277.

5. M. Davidson, G. Olafsson and G. Zhang, Laplace and Segal-Bargmann transforms on Hermitian symmetric spaces and orthogonal polynomials, J. Funct. Anal., 204 (2003), 157-195.

6. B. K. Driver and B. C. Hall, Yang-Mills theory and the Segal-Bargmann transform, Comm. Math. Phys., 201 (1999), 249-290.

7. L. D. Ėskin, Heat equation on Lie groups. (Russian) in: In Memoriam: N. G. Chebotarev, 113-132, Izdat. Kazan. Univ., Kazan, Russia, 1964.

8. J. Faraut, Formule de Gutzmer pour la complexification d'un espace riemannien symétrique, Harmonic analysis on complex homogeneous domains and Lie groups (Rome, 2001). Atti Accad. Naz. Lincei Cl. Sci. Fis. Mat. Natur. Rend. Lincei, (9) Mat. Appl., 13(3-4) (2002), 233-241.

9. J. Faraut, Analysis on the crown of a Riemannian symmetric space, Lie groups and symmetric spaces, Amer. Math. Soc. Transl. Ser. 2, 210, Amer. Math. Soc., Providence, RI, 2003, pp. 99-110.

10. J. Faraut, Espaces hilbertiens invariants de fonctions holomorphes. in: Analyse sur les groupes de Lie et théorie des représentations, (Kénitra, 1999), Sémin. Congr., 7, Soc. Math. France, Paris, 2003, pp. 101-167. 
11. G. B. Folland, Harmonic analysis in phase space, Annals of Mathematics Studies, 122. Princeton University Press, Princeton, NJ, 1989.

12. C. A. Florentino, P. Matias, J. M. Mourao and J. P. Nunes, Geometric quantization, complex structures, and the coherent state transform, J. Funct. Anal., 221 (2005), 303-322.

13. C. A. Florentino, P. Matias, J. M. Mourao and J. P. Nunes, On the BKS pairing for Kähler quantizations of the cotangent bundle of a Lie group, J. Funct. Anal., 234 (2006), 180-198.

14. R. Gangolli, Asymptotic behavior of spectra of compact quotients of certain symmetric spaces, Acta Math., 121 (1968), 151-192.

15. L. Gross and P. Malliavin, Hall's transform and the Segal-Bargmann map, In: Itô's stochastic calculus and probability theory, (M. Fukushima, N. Ikeda, H. Kunita and S. Watanabe, Eds.), Springer-Verlag, Berlin/New York, 1996, pp. 73-116.

16. V. Guillemin and M. B. Stenzel, Grauert tubes and the homogeneous Monge-Ampere equation, J. Differential Geom., 34 (1991), 561-570.

17. V. Guillemin and M. B. Stenzel, Grauert tubes and the homogeneous Monge-Ampere equation. II, J. Differential Geom., 35 (1992), 627-641.

18. B. C. Hall, The Segal-Bargmann "coherent state" transform for compact Lie groups, J. Funct. Anal., 122 (1994), 103-151.

19. B. C. Hall, The inverse Segal-Bargmann transform for compact Lie groups, J. Funct. Anal., 143 (1997), 98-116.

20. B. C. Hall, A new form of the Segal-Bargmann transform for Lie groups of compact type, Canad. J. Math., 51 (1999), 816-834.

21. B. C. Hall, Holomorphic methods in analysis and mathematical physics. in: First Summer School in Analysis and Mathematical Physics, S. Pérez-Esteva and C. Villegas-Blas, eds., Contemp. Math., 260, Amer. Math. Soc., Providence, RI, 2000, pp. 1-59.

22. B. C. Hall, Coherent states and the quantization of (1+1)-dimensional Yang-Mills theory, Rev. Math. Phys., 13 (2001), 1281-1305.

23. B. C. Hall, Harmonic analysis with respect to heat kernel measure, Bull. Amer. Math. Soc. (N.S.), 38 (2001), 43-78.

24. B. C. Hall, Bounds on the Segal-Bargmann transform of $L^{p}$ functions. J. Fourier Anal. Appl., 7(6) (2001), 553-569.

25. B. C. Hall, Geometric quantization and the generalized Segal-Bargmann transform for Lie groups of compact type, Comm. Math. Phys., 226 (2002), 233-268.

26. B. C. Hall, The Segal-Bargmann transform and the Gross ergodicity theorem. in: Finite and infinite dimensional analysis in honor of Leonard Gross, H.-H. Kuo and A. N. Sengupta, eds., Contemp. Math., 317, Amer. Math. Soc., Providence, RI, 2003, pp. 99-116. 
27. B. C. Hall, The range of the heat operator. in: The ubiquitous heat kernel, J. Jorgensen and L. Walling, eds., Contemp. Math., 398, Amer. Math. Soc., Providence, RI, 2006, pp. 203-231.

28. B. C. Hall and J. J. Mitchell, Coherent states on spheres, J. Math. Phys., 43 (2002), 1211-1236.

29. B. C. Hall and J. J. Mitchell, The Segal-Bargmann transform for noncompact symmetric spaces of the complex type, J. Funct. Anal., 227 (2005), 338-371.

30. B. C. Hall and J. J. Mitchell, Isometry formula for the Segal-Bargmann transform on a noncompact symmetric space of the complex type, J. Funct. Anal., 254 (2008), 1575-1600.

31. B. C. Hall and A. N. Sengupta, The Segal-Bargmann transform for path-groups, J. Funct. Anal., 152 (1998), 220-254.

32. S. Helgason, Differential geometry, Lie groups, and symmetric spaces, Corrected reprint of the 1978 original, Graduate Studies in Mathematics, 34. American Mathematical Society, Providence, RI, 2001.

33. S. Helgason, Groups and geometric analysis, Integral geometry, invariant differential operators, and spherical functions, Corrected reprint of the 1984 original. Mathematical Surveys and Monographs, 83. American Mathematical Society, Providence, RI, 2000.

34. S. Helgason, Geometric analysis on symmetric spaces, Mathematical Surveys and Monographs, 39. American Mathematical Society, Providence, RI, 1994.

35. J. Huebschmann, Kirillov's character formula, the holomorphic Peter-Weyl theorem, and the Blattner-Kostant-Sternberg pairing, J. Geom. Phys., 58 (2008), 833-848.

36. B. Krötz, G. Ólafsson and R. Stanton, The image of the heat kernel transform on Riemannian symmetric spaces of the non-compact type, Int. Math. Res. Not., 2005, 1307-1329.

37. B. Krötz and R. J. Stanton, Holomorpic extensions of representations: (I) automorphic functions, Ann. Math., 159 (2004), 641-724.

38. B. Krötz and R. J. Stanton, Holomorphic extension of representations: (II) geometry and harmonic analysis, Geom. Funct. Anal., 15 (2005), 190-245.

39. B. Krötz, S. Thangavelu and Y. Xu, The heat kernel transform for the Heisenberg group, J. Funct. Anal., 225 (2005), 301-336.

40. B. Krötz, S. Thangavelu and Y. Xu, Heat kernel transform for nilmanifolds associated to the Heisenberg group, Rev. Mat. Iberoam., 24 (2008), 243-266.

41. M. Lassalle, Series de Laurent des fonctions holomorphes dans la complexication d'un espace symetrique compact, Ann. Scient. École Norm. Sup., 11 (1978), 167210.

42. É. Leichtnam, F. Golse and M. B. Stenzel, Intrinsic microlocal analysis and inversion formulae for the heat equation on compact real-analytic Riemannian manifolds, Ann. Sci. École Norm. Sup., 29(4) (1996), 669-736. 
43. L. Lempert and R. Szóke, Global solutions of the homogeneous complex MongeAmpere equation and complex structures on the tangent bundle of Riemannian manifolds, Math. Ann., 290 (1991), 689-712.

44. E. Nelson, Analytic vectors, Ann. of Math., 70(2) (1959), 572-615.

45. G. Olafsson and B. Ørsted, Generalizations of the Bargmann transform. in: Lie theory and its applications in physics (Clausthal, 1995), World Sci. Publishing, River Edge, NJ, 1996, pp. 3-14.

46. G. Ólafsson and H. Schlichtkrull, The Segal-Bargmann transform for the heat equation associated with root systems, Adv. Math., 208 (2007), 422-437.

47. G. Olafsson and H. Schlichtkrull, Representation theory, Radon transform and the heat equation on a Riemannian symmetric space. in: Group representations, ergodic theory, and mathematical physics: a tribute to George W. Mackey, R. S. Doran, C. C. Moore and R. J. Zimmer, eds., Amer. Math. Soc., RI, 2008, pp. 315-344.

48. I. E. Segal, Mathematical problems of relativistic physics, Chap.VI, in: Proceedings of the Summer Seminar, Boulder, Colorado, 1960, Vol. II., M. Kac, ed., Lectures in Applied Mathematics, American Math. Soc., Providence, Rhode Island, 1963.

49. I. E. Segal, Mathematical characterization of the physical vacuum for a linear BoseEinstein field, Illinois J. Math., 6 (1962), 500-523

50. I. E. Segal, The complex-wave representation of the free Boson field. in: Topics in Functional Analysis, I. Gohberg and M. Kac, eds., Advances in Mathematics Supplementary Studies, Vol. 3, Academic Press, New York, 1978.

51. C. Sogge and S. Zelditch, Riemannian manifolds with maximal eigenfunction growth, Duke Math. J., 114 (2002), 387-437.

52. M. B. Stenzel, The Segal-Bargmann transform on a symmetric space of compact type, J. Funct. Anal., 165 (1999), 44-58.

53. M. B. Stenzel, An inversion formula for the Segal-Bargmann transform on a symmetric space of non-compact type, J. Funct. Anal., 240 (2006), 592-608.

54. R. Szbke, Complex structures on tangent bundles of Riemannian manifolds, Math. Ann., 291 (1991), 409-428.

55. R. Szóke, Adapted complex structures and geometric quantization, Nagoya Math. J., 154 (1999), 171-183.

56. R. Szbke, Involutive structures on the tangent bundle of symmetric spaces. Math. Ann., 319 (2001), 319-348.

57. A. Tyurin, Quantization, classical and quantum field theory and theta functions, With a foreword by Alexei Kokotov. CRM Monograph Series, 21. American Mathematical Society, Providence, RI, 2003.

58. H. Urakawa, The heat equation on compact Lie group, Osaka J. Math., 12 (1975), 285-297. 
59. K. K. Wren, Constrained quantisation and $\theta$-angles. II, Nuclear Phys. B., 521 (1998), 471-502.

Brian C. Hall

University of Notre Dame

Department of Mathematics

Notre Dame, IN 46556-4618

U.S.A.

E-mail: bhall@nd.edu

Jeffrey J. Mitchell

Robert Morris University

Department of Mathematics

6001 University Boulevard

Moon Township, PA 15108

U.S.A.

E-mail: mitchellj@rmu.edu 